\title{
Reconsiderando la introducción del maíz en el occidente de América del Sur
}

Reconsidérant l'introduction du maïs à l'ouest de l'Amérique du Sud Reconsidering the introduction of maize in the west of South America

John E. Staller y Robert G. Thompson

\section{(2) OpenEdition}

Journals

Edición electrónica

URL: http://journals.openedition.org/bifea/7337

DOI: $10.4000 /$ bifea. 7337

ISSN: 2076-5827

Editor

Institut Français d'Études Andines

Edición impresa

Fecha de publicación: 1 abril 2001

Paginación: 123-156

ISSN: 0303-7495

Referencia electrónica

John E. Staller y Robert G. Thompson, « Reconsiderando la introducción del maíz en el occidente de América del Sur », Bulletin de l'Institut français d'études andines [En línea], 30 (1) | 2001, Publicado el 08 abril 2000, consultado el 08 diciembre 2020. URL : http://journals.openedition.org/bifea/7337 ; DOI : https://doi.org/10.4000/bifea.7337

\section{(c) $(1)$}

Les contenus du Bulletin de l'Institut français d'études andines sont mis à disposition selon les termes de la licence Creative Commons Attribution - Pas d'Utilisation Commerciale - Pas de Modification 4.0 International. 


\title{
RECONSIDERANDO LA INTRODUCCIÓN DEL MAÍZ EN EL OCCIDENTE DE AMÉRICA DEL SUR
}

\author{
John E. STALLER*, Robert G. THOMPSON**
}

\begin{abstract}
Resumen
Numerosos investigadores en las ciencias sociales y naturales han discutido el origen y antigüedad del maíz en la región noroeste de América del Sur. En este artículo nos referimos a la evidencia disponible y a algunas de las teorías de los estudiosos sobre este tema. Presentamos además un nuevo método que permite establecer los usos que se le han dado al maíz, a través del estudio de los opalofitolitos encontrados en restos carbonizados procedentes de vasijas cerámicas de uso ritual. La excavación de una plataforma o montículo ceremonial en el sitio Valdivia de La Emerenciana ha permitido el hallazgo de numerosos rasgos arqueológicos, la mayor parte de los cuales son ofrendas rituales. Se analizaron restos carbonizados de las superficies interiores de dichas vasijas con el propósito de detectar la presencia de fitolitos de ópalo. Tres de las diez muestras analizadas procedentes de contextos confiables con fechados radiocarbónicos (calibrados) que abarcan entre 2203 y 1679 A.C. presentan fitolitos de maíz. Los restos de carbón de dos de las diez muestras fueron fechados con el método de aceleración de partículas (AMS) para establecer su cronología con mayor precisión. Se incorporan en este estudio datos complementarios obtenidos del análisis de isótopos de carbono de los esqueletos Valdivianos de contextos más tempranos de otros lugares de la costa, y cromatografía de papel para detectar aminoácidos. Asimismo, se consideran y discuten distintos aspectos vinculados con las asociaciones contextuales y los aspectos teóricos referidos a la difusión del maíz en el noroeste de Sudamérica. Nuestras conclusiones indican que el maíz fue introducido en la economía de subsistencia Valdivia en las últimas épocas de la secuencia, más como planta con valor ritual que económico.
\end{abstract}

Palabras claves: Noroeste de los Andes, paleobotánica, Ecuador, Valdivia, maíz.

${ }^{*}$ Department of Anthropology, University of Illinois - Chicago 1007 W. Harrison, Chicago, Illinois 60607 y Department of Anthropology, The Field Museum, Chicago, IL, 60605. E-mail: jstaller@uic.edu

*** Interdisciplinary Archaeological Studies, University of Minnesota, Minneapolis, Minnesota 55455. E-mail: rthompson@ @ tc.umn.edu 


\title{
RECONSIDÉRANT L'INTRODUCTION DU MAÏS À L'OUEST DE L'AMÉRIQUE DU SUD
}

\section{Résumé}

De nombreux chercheurs en sciences sociales et naturelles ont discuté sur l'origine et l'ancienneté du maïs au nord-ouest de l'Amérique du Sud. Dans cet article, nous nous référons aux preuves disponibles et à quelques unes des théories des chercheurs sur ce thème. Nous présentons, en outre, une nouvelle méthode permettant d'établir les usages donnés au maïs à travers l'étude des opalo-phytolithes retrouvés dans les restes carbonisés de céramiques à usage rituel. La fouille d'une plateforme ou monticule cérémoniel sur le site Valdivia de La Emerenciana a permis de trouver de nombreux restes archéologiques, dont une grande partie sont des offrandes rituelles. Des restes carbonisés des superficies intérieures de ces céramiques ont été analysés afin de détecter la présence de phytolithes d'opale. Sur les 10 échantillons analysés provenant de contextes fiables avec des datations radiocarboniques (calibrages) qui vont de 2203 a 1679 a.C., trois présentent des phytolithes de maïs. Les restes de charbon de 2 des 10 échantillons ont été datés grâce à la méthode d' accélération des particules (AMS) afin d'établir leur chronologie avec une plus grande précision.

En outre, on ajoute à cette étude des informations complémentaires obtenues de l'analyse des isotopoes de carbone des squelettes de l'époque Valdivia, de contextes plus anciens provenant d'autres endroits de la côte ; on utilise également du papier chromatographique pour détecter les acides aminés.

De même, nous considérons et discutons certains aspects líes aux associations contextuelles ainsi que les aspects théoriques concernant la diffusion du maïs dans le nord-ouest de l'Amérique du Sud. Nos conclusions indiquent que le maïs fut introduit dans l'économie de subsistance Valdivia au cours des derniers moments de la séquence, plutôt avec une valeur rituelle qu'économique.

Mots clés : Nord-ouest des Andes, paléo-botanique, Équateur, Valdivia, maïs.

\section{RECONSIDERING THE INTRODUCTION OF MAIZE IN THE WEST OF SOUTH AMERICA}

\begin{abstract}
A number of scholars in the social and natural sciences have debated the origin and antiquity of maize in northwestern South America. In this article we directly address some of the evidence and theories presented regarding this issue and present a new method for tracing the use of maize using opal phytolith assemblages from food residues in utilized ceramics. Archaeological excavations of a ceremonial platform mound at the Valdivia site at La Emerenciana have uncovered numerous archaeological features consisting primarily of ritual offerings. Carbon residues from the interior surfaces of excavated pottery contained in such features were analyzed for the presence of opal phytoliths. Three of the ten samples analyzed contain phytolith assemblages representative of maize cob chaff. The samples are from secure archaeological contexts in a layer radiocarbon dated to between 2203 and 1679 B.C. Carbon residues from two of the ten samples were AMS dated to more precisely establish their age. Complementary data recovered by paper chromatography of amino acids and carbon isotope analysis are incorporated into this study as were phytoliths removed from two of the four Valdivia burials from the La Emerenciana excavations. Contextual and theoretical issues as well as carbon isotope data from Valdivia skeletons from earlier contexts and from other regions of the coast are also reviewed regarding their significance to the initial spread of maize in northwestern South America. Our results indicate that maize was introduced into coastal Ecuador during the final portion of the
\end{abstract}


Valdivia culture sequence and that it may have been integrated into the subsistence economy as a ritual rather than an economic plant.

Key Words: NW Andes, paleobotany, Ecuador, Valdivia, maize.

\section{INTRODUCCIÓN}

La antigüedad de la introducción del cultivo del maíz en la parte occidental de Sudamérica es en la actualidad un tema de controversia. Existen dos propuestas al respecto: la que propone una introducción temprana, de ca. 7000 A.P. y la que sostiene una ocurrencia mucho más reciente, de ca. 4000-3500 A.P. La posición que considera la introducción temprana está principalmente basada en la evidencia de fitolitos publicada por Pearsall y Piperno (Pearsall, 1987; 1992a; 1992b; 1993; Pearsall \& Piperno, 1990; 1993; Piperno, 1984; 1985; 1990; 1991; 1994; Piperno et al., 1985).

La fecha propuesta de 7000 años de antigüedad para la introducción del maíz en Sudamérica ha sido criticada por algunos investigadores especializados en el estudio del origen y dispersión de este importante cultígeno. Estos estudiosos han planteado dudas principalmente de naturaleza contextual y metodológica (Bruhns, 1994; Fritz, 1994a; w994b; Smith, 1998; Rovner, 1999). Debe señalarse que en los fundamentos de la naturaleza de nuestros datos, está el hecho de que los conjuntos de fitolitos en depósitos carbonizados de residuos de comida hallados en los tiestos son difíciles si no imposibles de contaminar con otros fitolitos procedentes, sea de la matriz sedimentaria, sea de otros contextos, o de otros periodos de tiempo. Tal evidencia está asociada directamente con la olla o tiesto sobre el que se la encuentra. Por lo tanto, el propósito de despejar estas inquietudes nos llevó a tomar un enfoque multidisciplinario en nuestro estudio de la excavación de contextos arqueológicos que se asocian a la Fase Valdivia (3500-1500 A.C.) del sitio La Emerenciana, situado en la Provincia de El Oro, en la costa sud del Ecuador(Fig. 1). LaEmerenciana es un sitio significativo para la arqueología ecuatoriana porque es el centro ceremonial Valdiviano situado más al sur en la costa y donde el complejo de la cerámica Fase Jelí constituye en Sudamérica la ocurrencia más temprana documentada en el registro arqueológico, de material cerámico en formas de botella incluyendo botellas simples y asas estribos (Staller, 1992-1993; 1994; 1996; en prensa).

El análisis de residuos alimenticios proporciona un referente arqueológico seguro para el estudio de restos procedentes de sitios de Sudamérica, si tomamos en cuenta las dudas planteadas por los trabajos ya citados y nos centramos en los procesos culturales que se reflejan en el cambio en los hábitos culinarios. Como Roosevelt (1984: 8) ha observado

“.... no es lo mismo establecer la presencia de ciertas especies arqueológicamente, como lo es determinar el rol de la especie vegetal en la economía antigua."

La presencia de fitolitos de maíz en una muestra de residuos alimenticios nos habla directamente de su rol en la economía de subsistencia Valdivia. 


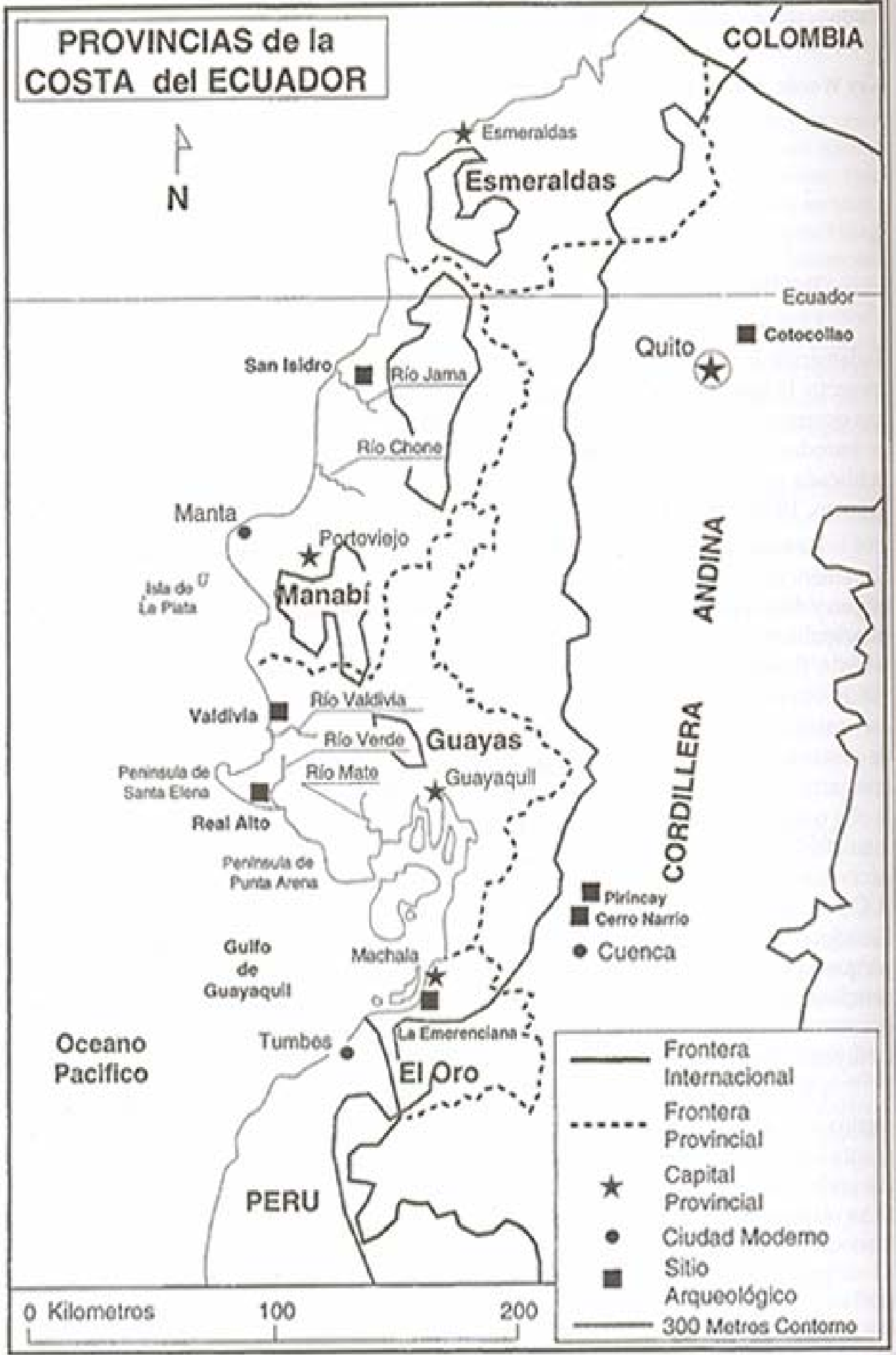

Fig. 1 - Provincias de la costa del Ecuador 
Si bien el conjunto de fitolitos puede reflejar la presencia o ausencia de maíz en una muestra de residuos alimenticios, los fitolitos por sí mismos no reflejan el porcentaje de maíz que aparece en dichos residuos. Hastorf \& DeNiro (1985) han aplicado análisis de isótopos de carbono para determinar la cantidad relativa de maíz en los residuos. Nosotros aplicamos este análisis a cinco muestras de residuos, pero lo complementamos con el de búsqueda de aminoácidos por medio de la cromatografía de papel. El análisis de fitolitos ha sido ya utilizado para detectar la presencia de maíz en los residuos alimenticios (Thompson \& Mulholland, 1994). Los marlos producen en abundancia fitolitos del tipo rondel en el pericarpio, en el marlo y en las estructuras accesorias de la espiga. Como resultado del procesamiento del maíz para consumo, se obtiene un conjunto reconocible de fitolitos incorporados a los alimentos, y consecuentemente a los residuos carbonizados adheridos a la cerámica. La producción de fitolitos rondel en el marlo fue lo suficientemente diferente de la encontrada en residuos alimenticios con presencia de gramíneas del área de Dakota del Norte como para ser por sí sola una prueba del uso del maíz. Si bien el maíz presente actualmente en las Grandes Planicies y el Noreste de Norteamérica es genéticamente divergente y único (Doebley et al., 1986), este análisis indica que la misma técnica puede ser aplicada a formas de otras regiones. Nuestra evidencia constituye un desafío a las interpretaciones previas acerca de la importancia del maíz para la economía de subsistencia Valdivia temprana (ver por ej. Pearsall, 1978; 1993; Pearsall \& Piperno, 1990; Zevallos, 1971; Zevallos et al., 1977). Las evidencias arqueológicas, etnobotánicas e isotópicas presentadas en este análisis demuestran que la introducción inicial del maíz en la costa del Ecuador se dio ca. 2200 a 1950 A.C. También consideramos cuestiones más amplias relacionadas con el origen de la agricultura de maíz en los Andes.

\section{1. ÓPALOFITOLITOS}

Los ópalofitolitos son restos paleobotánicos de microfósiles de plantas compuestos por sílice amorfo exudado por las mismas. En la naturaleza las especies vegetales toman ácido monosilíceo del suelo cuando obtienen nutrientes por medio de las raíces. Mientras la mayoría de los nutrientes son incorporados en compuestos orgánicos usados por la misma planta, el sílice no lo es. El sílice es depositado de diferentes formas por las plantas entre sus células. Aunque la razón del depósito de esos cuerpos silíceos no se comprende bien aún, el depósito en sí mismo está bien documentado. En una monografía fundamental sobre gramíneas Metcalf (1960) señaló la ubicuidad de los fitolitos en esta familia. Más recientemente otros estudiosos (Bozarth, 1987; Pearsall, 1978; 1982; 1992a; 1993; Piperno, 1988; Rovner, 1971; 1983) han señalado que los fitolitos pueden ser producidos por una gran variedad de plantas. Los fitolitos han demostrado poseer en diferentes grados, utilidad taxonómica en la identificación de las especies vegetales de las cuales provienen (Pearsall, 1982; 1989; 1992a; 1992b; 1993; Piperno, 1984; 1988; Pearsall \& Piperno, 1993; Piperno \& Pearsall, 1993).

Los ópalofitolitos son muy durables y permanecen morfológicamente intactos hasta mucho después de su depósito en el suelo. En efecto, se han encontrado ópalofitolitos en depósitos del Mioceno (Piperno, 1988). Su potencial como instrumento para la investigación arqueológica de cualquier período de ocupación humana es obvio. De más importancia para este estudio es el hecho de que los ópalofitolitos soportan sin 
alterarse el calor de la cocción. De hecho uno de los primeros métodos para recuperar fitolitos llamado "secado de cenizas" consistía en incinerar la porción de la planta a temperaturas que excedían los $500^{\circ} \mathrm{C}$ (Rovner, 1983).

La mayor parte de las investigaciones previas se ha relacionado con el desarrollo de taxonomías en fitolitos (Rovner, 1983) como indicador de ambientes del pasado (Rapp \& Mulholland, 1992). El sistema taxonómico está basado en la morfología tridimensional de los ópalofitolitos desarrollada por Mulholland \& Rapp (1992). Aplicamos este sistema a los fitolitos recuperados descritos en nuestro análisis. En particular serán identificados los cuerpos silíceos asociados con gramíneas.

La técnica desarrollada por Pearsall y Piperno (Pearsall, 1978; 1982; 1994b; Piperno, 1984; 1988; Pearsall \& Piperno, 1993: 17-18; Piperno \& Pearsall, 1993) que comprende el uso de la morfología tridimensional, detecta la presencia de maíz en conjuntos de fitolitos de muestras de sedimentos en sitios arqueológicos (Smith, 1998). La tipología está basada en la identificación de las formas producidas únicamente por el maíz. Su foco son las cruces extra largas, generalmente encontradas en las hojas de gramíneas (Pearsall \& Piperno, 1993: 14-15, Tablas 1-4; Piperno \& Pearsall, 1993:347355, Tabla 7).

Nuestro análisis se basa en la producción de fitolitos de maíz en el marlo y estructuras accesorias. El material remanente de las hojas (la "chala" que envuelve el marlo es una hoja modificada) se encuentra habitualmente ausente en los residuos de alimentos. En el procesamiento del maíz para la alimentación, las porciones del marlo y por lo tanto los cuerpos silíceos que produce, generalmente están incorporados. Los cuerpos silíceos considerados individualmente no son identificables como propios del maíz. Sin embargo los producidos por el marlo son fácilmente reconocibles cuando forman conjuntos. La Tab. 1 enumera las ventajas de la detección de la presencia de maíz mediante la utilización de conjuntos de cuerpos silíceos del marlo y la Tab. 2 proporciona los tipos más comunes de ópalo fitolitos observados hasta la fecha. Estos tipos incluyen células que no son particularmente útiles para taxonomía, de la misma forma que algunas que sí lo son. La categoría 7 es un indicador de gramíneas, que contienen células de sílice especializadas. La Tab. 3 enumera las subdivisiones generales de cuerpos silíceos de gramíneas utilizados en este estudio.

\section{FITOLITOS A PARTIR DE ISOTÓPICOS DE LÍPIDOS Y RESIDUOS ALIMENTICIOS}

Una investigación reciente llevada a cabo por Thompson \& Mulholland (1994) ha demostrado que la presencia de maíz puede ser determinada en ópalofitolitos recuperados en los residuos alimenticios de vasijas cerámicas (ver también Thompson, 1986; 1987a; 1987b). Cuando el maíz es preparado como comida, fragmentos de las estructuras anexas al marlo son incorporados al alimento. Si bien estos residuos comprenden sólo una pequeña fracción del alimento resultante por unidad de peso, las células de sílice forman una porción sustancial de los residuos alimenticios debido a la durabilidad de la estructura de dióxido de silicona. Por lo tanto, la evidencia a partir de los residuos de alimentos y del tártaro dental se refiere directamente a las relaciones contextuales previamente mencionadas. 
Tab. 1 - Ventajas del conjunto de desechos de marlos y estructuras asociadas.

\begin{tabular}{|cl|}
\hline \multicolumn{1}{|c|}{$\begin{array}{c}\text { Ventajas en el rastreo de la presencia de maíz con análisis de fitolitos } \\
\text { basados en la presencia de conjuntos de desechos de marlos y } \\
\text { estructuras asociadas }\end{array}$} \\
\hline \multicolumn{1}{|c|}{ Autor } & \multicolumn{1}{c|}{ Ventaja } \\
\hline Dorweiler \& Doebley (1997), & $\begin{array}{l}\text { El control genético del depósito de sílice en el } \\
\text { choclo (alvéolos y hojas de chala) de la mazorca } \\
\text { horweiler } \text { et al. (1993) }\end{array}$ \\
$\begin{array}{l}\text { hido demostrado, incluyendo variación } \\
\text { subespecífica en el depósito } \\
\text { Se ha demostrado que la harina de maíz } \\
\text { (resultante del procesamiento del maíz) } \\
\text { contiene abundantes cuerpos silíceos } \\
\text { procedentes del marloy estructuras accesorias. } \\
\text { Residuos de marlos de maíz se encuentran } \\
\text { entre los residuos alimenticios, lo cual resulta } \\
\text { en un contexto cultural incuestionable }\end{array}$ \\
\hline
\end{tabular}

Nota: Todas las referencias estuvieron originalmente en inglés. La traducción es nuestra.

Tab. 2 - Categorías de fitolitos.

1. Trichomas - Pelos y papilae. Base esférica a ovoide con un extremo cónico.

2. Stomata - Células protectoras y/o subsidiarias. El conjunto entero es de forma ovoide. Las células protectoras tienen forma de tubo telefónico. Las células subsidiarias son ovoides a triangulares.

3. Células vesiculiformes - Células epidérmicas agrandadas, de paredes delgadas. Formas en arquitrabe.

4. Células epidérmicas basales - Células epidérmicas no especializadas. Varias formas prismáticas cuadrangulares con bordes engranados. Delgadas.

5. Varas - Fibras, escleridias, células de xilema y otras células de formas cilíndricas.

6. Células prismáticas grandes - Rectangulares/cuadradas. Prisma cúbico a rectangular. Más espesas que las células basales o cuerpos silíceos.

7. Cuerpos silíceos - Fitolitos de células especializadas en la acumulación de sílice. Conos, pirámides, prismas rectangulares y cilindros truncados a biselados. Por lo menos una faceta ancha (base) está presente. Nótese que, aunque los cuerpos silíceos son equiparados con células cortas en los textos botánicos, algunos cuerpos silíceos muy fuertes están incluidos aquí con los más cortos. Los cuerpos silíceos largos están consistentemente silicificados y se asemejan con los otros cuerpos silíceos en la textura superficial (a diferencia de las células basales que se hallan silicificadas). Por estas razones, las células más largas se incluyen aquí.

(Según Mulholland \& Rapp, 1992)

Nota: Las categorías 1-6 no siempre están silicificadas en las gramíneas. La categoría 7 está consistentemente silicificada (de hecho es una característica identificatoria). 
Tab. 3 - Formas de cuerpo silíceo de gramíneas.

I. El cuerpo va de una caja rectangular a una pirámide truncada o biselada; la sección en la base es aproximadamente rectangular o cuadrada o en forma de otro polígono (la base puede tener lóbulos pero el contorno general es un polígono); el extremo es una faceta chata a levemente cóncava o convexa o con borde(s) elevado(s).

A. Nolobado: lados de la base carecen de lóbulos definidos

1. Base de 3 lados

TRIÁNGULO

2. Base de 4 lados RECTÁNGULO

3. Base de 5 lados PENTÁGONO

B. Lobado: lados de la base con lóbulos definidos

1. Diámetros mínimos de la base aproximadamente iguales

CRUZ

2. Diámetros mínimos de las bases desiguales

a. Bilobado: máximo de 2 lóbulos por lado

1. Proporción diáfisis/lóbulo $>2 / 3$

SINUOSO

2. Proporción diáfisis/lóbulo $<2 / 3$ DUMBBELL

(PESA)

b. Polilobado: más de 2 lóbulos por lado

1. Proporción diáfisis/lóbulo $>2 / 3$

SINUOSO

2. Proporción diáfisis/lóbulo $<2 / 3$

DUMBBELL

(PESA)

II. El cuerpo va de un cilindro corto a un cono truncado o biselado; la sección de la base es aproximadamente oval, circular o tiene otra forma curvada (la base puede tener segmentos cóncavos o chatos pero el contorno general es una forma curva); el extremo es una faceta chata a levemente cóncava o convexa o con borde(s) elevado(s).
A. Entero: bordes de la base todos convexos
RONDEL
B. Achatados: algunos bordes de la base rectos
RONDEL
C. Indentado: algunos bordes de la base cóncavos RONDEL

III. El cuerpo tiene forma de montura; la sección del extremo o ambos, extremo y base, tiene dos bordes convexos opuestos que sobresalen de la superficie facetal y dos bordes inferiores opuestos que son generalmente cóncavos; el extremo es cóncavo.
A. Tabular: extremo y base de la misma forma y tamaño MONTURA
B. Plateau: extremo más chico o de forma diferente
MONTURA
C. Borde: el extremo es un borde
MONTURA

(Según Mulholland \& Rapp, 1992)

El análisis presentado en este estudio está basado en una técnica que abarca el examen de un conjunto completo de fitolitos encontrado en residuos y en tártaro dental. Con tan robusta base de datos, tratamos las relaciones estadísticas y contextuales que se presentan en la tabla uno (Dolittle \& Fredrick, 1991; Fritz, 1994a; 1994b; Smith, 1998). Estos conjuntos están formados por fitolitos de marlo en los cuales el depósito de sílice difiere en una base subespecífica (Dorweiler \& Doebley, 1997; Dorweiler et al., 1993). 
Dos de las muestras de residuos orgánicos fueron datadas directamente por espectrometría de aceleración de masa (AMS) ya que sólo pequeñas cantidades de carbón eran requeridas para el procesamiento. Cuando se lleva a cabo una datación de este tipo, los resultados del análisis de carbón isotópico son también proporcionados por rutina.

Los niveles de isótopos de carbono han sido reconocidos desde hace tiempo como un método para detectar la presencia y abundancia de especies vegetales C4 tales como el maíz (DeNiro, 1987; Hastorf, 1994).

Una investigación reciente sobre fitolitos ha logrado proporcionar líneas directas de evidencia respecto a la función de las vasijas. Como muchos otros artefactos materiales, la cerámica puede reflejar su función en el pasado de muchas maneras. Braun (1983; 1987) notó cambios en la forma de las vasijas y en el engrosamiento de las paredes asociados con cambios en la función de tales recipientes. Las vasijas de almacenamiento y ollas de cocina frecuentemente proporcionan residuos orgánicos en la superficie de sus tiestos. Hastorf \& DeNiro (1984) han detectado la presencia de maíz en la costa del Perú a través del análisis de residuos alimenticios utilizando para ello isótopos de carbono. También se han utilizado lípidos para analizar residuos alimenticios, y detectar la presencia de maíz (Heron et al., 1991; Letts et al., 1994). En la primera parte de nuestro siglo, se encontró arroz en residuos alimenticios hallados en cerámica China de cinco mil años de antigüedad (Edman \& Soderberg, 1929). Como ilustran estos ejemplos, el análisis de residuos alimenticios ha sido conducido de distintas formas, con el objeto de establecer los usos de la cerámica y la existencia de especies vegetales en contextos arqueológicos. Este estudio abarca la identificación de restos silíceos de especies vegetales, u ópalofitolitos, en los residuos alimenticios hallados en tiestos de cerámica procedentes de excavaciones. Los ópalofitolitos pueden ser útiles taxonómicamente en la identificación de las especies vegetales que los producen. Thompson (1986) detectó la presencia de maíz en residuos alimenticios usando esta técnica (ver también Thompson \& Dogan, 1987). Más recientemente, Thompson \& Mulholland (1994) tuvieron éxito al comprobar el uso del maíz en cerámica del este de Dakota del Norte empleando la técnica descrita en este estudio. Sin embargo, a pesar de la utilización de tal análisis en el estudio del origen del maíz en Sudamérica, ha habido poca aplicación de esta técnica a la cultura material precolombina.

Este estudio proporciona un contraste ideal en enfoques paradigmáticos entre el análisis de sedimentos y el análisis de residuos de alimentos. Los residuos de alimentos proporcionan un contexto único para el análisis de fitolitos, y requieren de un enfoque diferente del que ha sido utilizado tradicionalmente en el análisis de sedimento. El enfoque paradigmático del análisis de conjuntos de fitolitos a partir de sedimentos es necesariamente paleoambiental.

La identificación de fitolitos obtenidos a partir de sedimentos arqueológicos presenta una enigma contextual porque cualquier planta productora de sílice en la vecindad del sitio debe ser considerada como fuente potencial del conjunto observado. Por lo tanto, todas las especies vegetales del área del sitio durante el tiempo de la ocupación deben ser reconstruidas, del mismo modo que los cambios temporales en la vegetación. El paradigma del análisis de residuos alimenticios es la reconstrucción de la dieta del pasado. En los residuos alimenticios (incluyendo el tártaro dental) se puede 
razonablemente esperar encontrar sólo plantas alimenticias, reduciéndose mucho el número de especies vegetales que necesitan ser examinadas en un estudio dado. El foco sobre plantas utilizadas proporciona la oportunidad de explorar un grupo más limitado de fitolitos en mayor detalle.

\section{EVIDENCIA DE MAÍZ EN EL FORMATIVO DEL ECUADOR Y PERÚ}

Meggers y Evans sostuvieron en sus últimas publicaciones sobre el tema que el maíz domesticado del Ecuador fue introducido desde Mesoamérica ca. 1500 A.C. Consideran además que fue un elemento básico en la costa durante la Fase Chorrera del Período Formativo Tardío (ca. 1500-300 A.C.) (Meggers, 1966: 55, 64; Evans \& Meggers, 1966: 203-204; Meggers et al., 1965). Más al sur en los desiertos de la costa peruana, un cuerpo importante de evidencia macrobotánica, etnobotánica y arqueológica de otras regiones ha contribuido también a sostener la introducción tardía (después de 1500 A.C.) del maíz (ver por ej., Bird, 1978: 92; 1979: 53-54; 1984: 43, 49; 1990: 828; Bird \& Bird, 1980: 330). Burger y van der Merwe (1990) apoyan esta hipótesis basándose en la presencia de isótopos estables de carbono a partir del análisis de colágeno óseo de ocho esqueletos del Período --Horizonte Temprano, Período Inicial y Período Precerámico Tardío, derivados respectivamente de contextos de Chavín de Huántar (ca. 850-200 A.C.) y en el sitio de Huaricoto asociado con la Fase Precerámica Chaukayan Tardía (ca. 2200 A.C.). Llegan a la conclusión de que hubo poco cambio en el consumo de gramíneas $\mathrm{C} 4$ en los contextos de Huaricoto, y en los contextos de la parte más tardía del Período Inicial/Período de Horizonte Temprano en Chavín de Huántar.

Una de las principales conclusiones obtenidas es que el maíz fue un producto agrícola de segundo orden en la dieta de las tierras altas del norte entre el 2200 y el 200 A.C., y que no parece haber sido un elemento catalizador para el desarrollo de la civilización en las tierras altas de Perú (Burger \& van der Merwe, 1990: 91). Tal estimación está en un todo de acuerdo con la mayoría de las fechas asociadas con sitios precerámicos tardíos en la costa norte (Bonavia \& Grobman, 1989a; 1989b). En sitios prehispánicos del Perú la preservación permite obtener evidencia macrobotánica directa de la presencia del maíz. En sitios precerámicos como el sitio Los Gavilanes situado a la vera del río Chicama, Bonavia \& Grobman (1989a, tabla 2) han observado evidencia de restos de varias partes del maíz incluyendo más de cien mazorcas completas. Ellos han presentado seis fechados radiocarbónicos y un fechado por termoluminiscencia (Bonavia \& Grobman, 1989a, Tabla 1), y todos los fechados radiocarbónicos concuerdan con las estimaciones basadas en la evidencia presentada en este análisis. Según los datos presentados en este estudio, y la evidencia del análisis isotópico realizado sobre más de cincuenta esqueletos Valdivianos de la costa ecuatoriana, se hace evidente que el maíz fue inicialmente introducido en algún momento entre 2200-1850 A.C. La evidencia de la costa peruana implica intercambio de maíz entre el Perú y la costa ecuatoriana que empezó simultáneamente con su introducción en la costa del Ecuador. Además, el maíz parece tener una larga historia como fuente secundaria de recursos alimenticios en las tierras altas del Perú antes 200 A.C., y también en la costa norte y central del Perú antes por lo menos 1000 A.C. 
Nuestros resultados, basados en métodos de análisis como el de fitolitos, aplicados directamente a residuos alimenticios, indican la presencia de maíz en el sitio de La Emerenciana recién inicialmente entre 2200 y 1950 fechas calibradas, un poco antes de los tiempos 1850-1500 A.C. o Valdivia VII y VIII en la fase ocho de la sequencia cerámica reportada por Hill (1972-1974). Estos resultados están en acuerdo con una evidencia esqueletal similarque ha sido presentada para poblaciones prehispánicas de la costa del Ecuador. El análisis de isótopos sobre cincuenta esqueletos de varios sitios Valdivianos de los contextos de las Fases I-III condujo a van der Merwe et al. (1993: 81) a concluir que, “... ni el maíz ni los alimentos marinos jugaron un papel importante en la dieta Valdivia temprana, y que lo mismo puede decirse para la Fase 3 Valdivia en Real Alto.” Más aún, enfatizan que ni el maíz ni los alimentos marinos eran particularmente importantes durante las Fases Valdivia tempranas (I-III), sugiriéndonos poner la atención a las explicaciones que destacan la importancia de la selva como base para la subsistencia (van der Merwe et al., 1993).

La más reciente evidencia paleobotánica y de isótopos de carbono nos indica que la economía Valdivia era heterogénea, con estrategias de explotación del ambiente que incluían la recolección de plantas silvestres estacionales, caza de mamíferos terrestres y aves, explotación de recursos marítimos y cultivo a pequeña escala (Pearsall, 1992a: 195-197; 1993: 110-122; Pearsall \& Piperno, 1990: 335). Sin embargo, a pesar de no haberse comprobado que el maíz fuera componente significativo de la subsistencia regional, Pearsall \& Piperno (1990: 335) concluyen que existe evidencia de una "intensificación" de su agricultura en la costa de las tierras bajas del Ecuador ca. 3500 A.P. La afirmación de que el maíz era cultivado intensivamente o que era un componente central en la economía de subsistencia Valdivia temprana (Lathrap et al., 1975: 19-22; Lathrap et al., 1977: 6-7; Zevallos, 1971: 17-20; Zevallos et al., 1977), ha sido invalidada por evidencia más reciente.

Diametralmente opuesto al ajustado concepto del cultivo de maíz temprano en Sudamérica, Smith (1998) y Fritz (1994a; 1994b) han proporcionado recientemente argumentos convincentes de una introducción mucho más tardía de la agricultura del maíz en Sudamérica. La idea fundamental de este punto de vista opuesto es que el maíz fue originalmente domesticado en una sola área del valle central del Río Balsas en el occidente de México. La teoría de un único origen es sostenida por el trabajo reciente en las relaciones genéticas de Zea (Doebley, 1990; Benz, 1998; Benz \& Iltis, 1990; Benz \& Long, 2000). En este esquema alternativo, el maíz es domesticado en el valle central del Río Balsas del sudeste de México en algún momento después de 7000 años atrás, y se expande en el continente sur en una fecha mucho más tardía (Smith, 1997; 1998; Benz \& Long, 2000 ).

Los arqueólogos que estudian la relación entre el maíz y el cambio cultural en Sudamérica han tenido también dificultades en la reconciliación del dilema cronológico proporcionado por los datos de la costa del Ecuador. En una síntesis reciente de la arqueología, Bruhns (1994: 95) concluye en un capítulo adecuadamente titulado "El Problema del Maíz” que el maíz en Sudamérica antes de los 6000 años atrás, y un solo y casi contemporáneo origen mexicano no pueden reconciliarse. 


\section{CUESTIONES ACERCA DE UNA INTRODUCCIÓN TEMPRANA DEL MAÍZ}

Varios estudios paleobotánicos recientes han identificado fitolitos de maíz a partir de sedimentos en una serie de sitios de los Períodos Precerámico y Cerámico Temprano como así también en núcleos de lagos en diferentes regiones de tierras bajas de los neotrópicos (ver Bush et al., 1989; Pearsall, 1987; 1992a; 1992b; 1993; Pearsall \& Piperno, 1990; Piperno \& Pearsall, 1993; Piperno, 1984; 1985; 1990; 1991; Piperno et al., 1985). Las interpretaciones y conclusiones de estos estudios paleobotánicos indican que la presencia más temprana de fitolitos de maíz en la costa del Ecuador se da en los sedimentos datados que pueden retrotraerse tan atrás como el 7000 A.P. Más aún, se afirma que dos clases diferentes eran utilizadas ca. 5200 A.P. (Pearsall, 1993: 112, 122; Pearsall \& Piperno, 1990: tabla 3; Piperno, 1990: 665; 1991: 173).

Las fechas tempranas para la agricultura del maíz dadas a conocer por Pearsall y Piperno no han sido aceptadas por los especialistas en la domesticación del maíz. Estos estudiosos sostienen principalmente que las asociaciones entre los sedimentos y los contextos arqueológicos son muy problemáticas (Doolittle \& Fredrick, 1991; Fritz, 1994a; 1994b; Smith, 1997; 1998). Algunos de los temas tratados por estos investigadores respecto a la capacidad del análisis de fitolitos para detectar la presencia de maíz son presentadas como citas directas en la Tabla 4.

Las críticas recientes a la idea de una introducción temprana del maíz en Sudamérica han dado lugar a interrogantes acerca de la validez de los ópalofitolitos recuperados en contextos arqueológicos y núcleos de lagos (Fritz, 1994a; 1994b; Smith, 1998). Smith (1998: 158) ha señalado la fragilidad estadística de los grandes fitolitos en forma de cruz descritos por Piperno (1984; Piperno \& Pearsall, 1993), y considera que las conclusiones de estos autores son cuanto más no significativas. Fritz (1994a; 1994b) ha detallado la naturaleza contextual problemática del maíz temprano en el Período Precerámico Tardío Las Vegas, material Valdivia y el núcleo lacustre Ayauach en la selva tropical de SE de Ecuador. Esta investigadora se muestra cautelosa especialmente en lo que respecta a la perturbación post depósito, y se hace eco de la preocupación de Smith respecto a la recuperación insuficiente. En base a eso, concluye que el maíz está presente tanto en los contextos arqueológicos como en los de núcleo lacustre. Recientemente algunos estudiosos (Doolittle \& Fredrick, 1991; Rovner, 1995; Rovner \& Russ, 1992; Russ \& Rovner, 1989) han establecido que la técnica de la cruz grande para identificar fitolitos de maíz no puede ser repetida con éxito. En Doolittle \& Fredrick (1991), Iltis señala que los conjuntos de fitolitos procedentes de hojas y de tallos de Zea mais son indistinguibles de los de Teosinte. Un trabajo reciente de Doebley et al. (1997) demuestra que el cambio genético en los tallos y hojas de la planta de maíz se relaciona con la evolución de la dominancia apical, y una pérdida de las yemas. Hasta el momento, las investigaciones que buscan confirmar cualquier efecto de este cambio en la depositación de sílice no han sido promisorias. Rovner (1999; comunicación personal) informa del hallazgo de fitolitos de tipo maíz en niveles estratigraficos precerámicos en un sitio del estado de Carolina del Norte(EE.UU.), procedentes de niveles datados desde 7000 AP y por lo menos 3000 años antes del arribo de los primeros pobladores en el sitio. El maíz es obviamente imposible en este contexto y estos resultados demuestran que los 
Tab. 4 - Problemas de análisis de fitolitos basados en la presencia de cruces extra grandes para el rastreo de la presencia del maíz.

\begin{tabular}{|l|l|}
\hline Doolittle \& Frederick & $\begin{array}{l}\text { No duplicado de la variación en la producción de } \\
\text { fitolitos en la hojas de maíz }\end{array}$ \\
\hline $\begin{array}{l}\text { Iltis in: Doolittle \& } \\
\text { Frederick (1991) }\end{array}$ & $\begin{array}{l}\text { Ausencia de una base genética o morfológica para la } \\
\text { variación en los fitolitos entre las hojas de maíz y } \\
\text { teosinte }\end{array}$ \\
\hline Fritz (1994b) & $\begin{array}{l}\text { Asociación contextual de las muestras sedimentarias } \\
\text { de sitios arqueológicos Valdivia y Vegas. "Posición } \\
\text { estratigráfica de fitolito único de maíz vs. el fechado } \\
\text { usado en la interpretación del núcleo del lago Ayuach." }\end{array}$ \\
\hline Smith (1998: 159) & $\begin{array}{l}\text { "Fragilidad estadística de los datos de fitolitos usados } \\
\text { por Piperno \& Pearsall(1990). Si el tamaño promediode } \\
\text { las cruces del tipo I, tal como se los mide en un } \\
\text { microscopio de luz, fuera reducido en solamente 0,1-0,4 } \\
\text { micrones, las cinco muestras de maíz (sin forma) ya no } \\
\text { se clasificarían como de maíz. Esto no parece ser una } \\
\text { evidencia muy fuerte para la presencia de maíz." }\end{array}$ \\
\hline
\end{tabular}

Nota: Todas las referencias estuvieron originalmente en inglés. La traducción es nuestra.

conjuntos de cuerpos cruciformes utilizados por Pearsall y Piperno para identificar la presencia de maíz son también producidos por hierbas silvestres. Mulholland (1990) ha demostrado que la metodología de identificación de Pearsall y Piperno puede llevar a identificar un pasto silvestre totalmente no relacionado como Arunda donax cuyos fitolitos no son realmente los mismos que se hallan en el maíz.

\section{TÉCNICAS DE LABORATORIO}

Cada una de las categorías de cuerpos silíceos descritos anteriormente pueden ser divididos en subcategorías mediante el análisis más detallado de los rasgos observables con el microscopio. Mulholland \& Rapp (1992) han publicado un análisis detallado de estos grupos. Recientemente Thompson revisó las subcategorías de rondels algo diferentes de aquellas publicadas por Mulholland \& Rapp (1992). El análisis de los conjuntos de fitolitos recuperados del sitio La Emerenciana está basado en algunas de estas subcategorías.

Observadas desde arriba, estas subcategorías de fitolitos rondel son aproximadamente circulares a ovales en visión plana. La forma tridimensional parece 
la de un cilindro corto, algo estrangulado en el medio (Fig. 2). Las revisiones taxonómicas en la clasificación descrita más adelante fueron hechas con la intención de incorporar mejor la morfología tridimensional, y se focalizaron en la forma de las caras del cilindro.

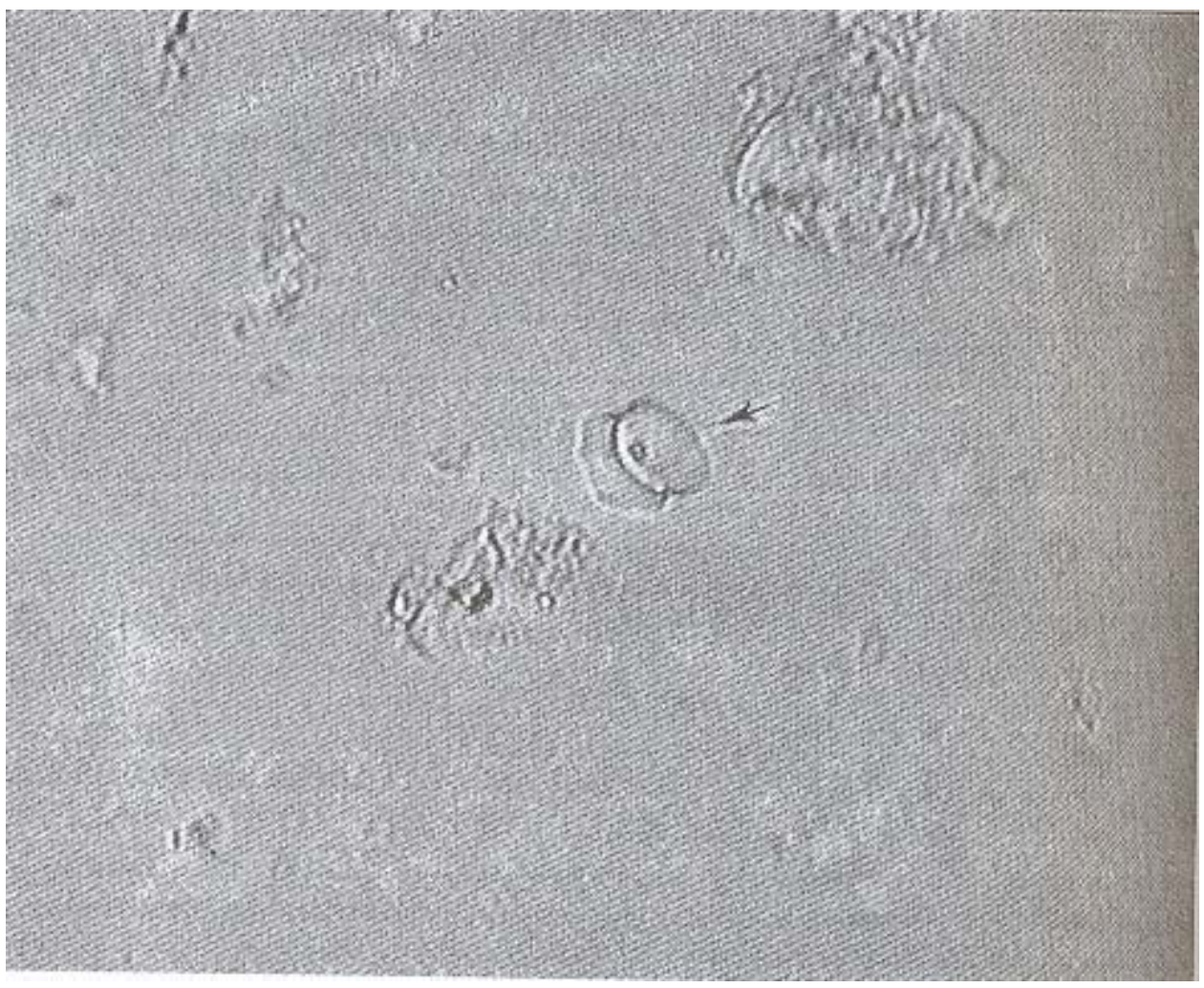

Fig. 2 - Forma rondel inclinado mostrando el cuerpo restringido, en carretel, Magnificación original 400X. Recuperado en residuos alimenticios de La Emerenciana.

Con el microscopio de luz, la cara más pequeña de un rondel parece más robusta, y es definida como la cara gruesa. Esta cara puede ser descrita como entera o con muescas, y el número de muescas puede ser contabilizado. La cara opuesta no es tan robusta, y se describe como la cara delgada. Esta cara puede también ser entera, o indentada, y el número de indentaciones se puede contabilizar (Fig. 3).

Cuando se cuentan los fitolitos recuperados en La Emerenciana, los rondels en la cara son descritos ya como enteros o denticulados, y con muescas o sin ellas, cuando tomamos en cuenta la cara espesa. Otras formas, tales como bilobabos o sinuosos son siomplemente contabilizados. 


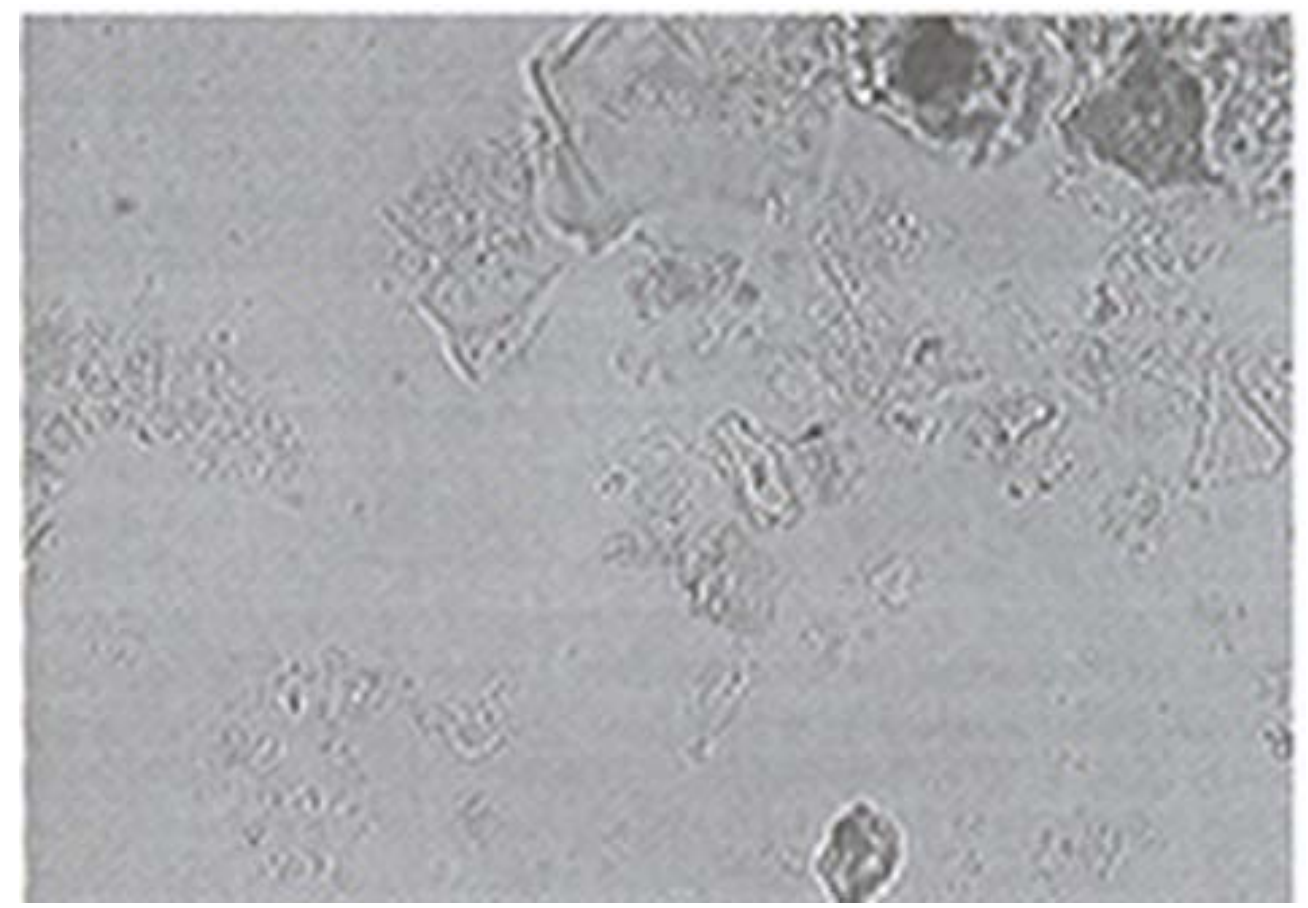

Fig. 3 - Rondel indentado-con muesca en vista planar. Maginificación original 400X. Colección comparativa (fitolitoteca?).

\section{FECHADO CON AMS Y ANÁLISIS DE ISÓTOPOS DE CARBONOS}

El análisis de isótopos de carbono ha demostrado ser útil en el reconocimiento de restos de diferentes tipos de gramíneas que se encuentran representados en el suelo humífero de las Grandes Planicies de Norteamérica (Kelly et al,,1991) y en los residuos alimenticios (Hastorf \& DeNiro, 1984) recuperados en el Valle del Mantaro en Perú. Las gramíneas funcionan de diferentes formas y acumulan diferencialmente ${ }^{13} \mathrm{C}$ de la atomósfera. Después de la muerte de la cantidad de ${ }^{13} \mathrm{C}$ se deteriora en una proporción previsible. El fechado por AMS permite que las muestras de residuos alimenticios sean fechados directamente, ya que sólo se requiere una pequeña cantidad de carbón para el análisis. Además, actualmente, cuando quiera que el fechado por AMS se lleve a cabo, el análisis de isótopos de carbono $\left({ }^{13} \mathrm{C} /{ }^{12} \mathrm{C}\right)$ se realiza por rutina y se proporciona conjuntamente con el fechado radiocarbónico. Los dos tipos principales de camino en que las plantas realizan su ciclo metabólico para la fijación del carbono se conocen como C3 y C4. Las plantas C3 acumulan relativamente menos ${ }^{13} \mathrm{C}$ durante sus vidas que las plantas C4. La cantidad de ${ }^{13} \mathrm{C}$ pueden medirse aún luego de la carbonización de los restos vegetales, y no es afectada por la combustión (DeNiro, 1987).

La Emerenciana está ubicada a 2 km de la marea bajo la línea de costa activa (Staller, 1994:204-204). El maíz es una planta C4; sin embargo la mayoría de las plantas usadas como alimento en la región son C3. El estudio intensivo de las plantas carbonizadas de la región sería necesario para obtener una estimación de los porcentajes del maíz en los residuos alimenticios. La abundancia relativa de ${ }^{13} \mathrm{C}$ en una muestra de residuo alimenticio puede ser medida contra las otras muestras, proporcionando así una medida relativa del uso de las plantas C4/C3. 


\section{CONSIDERACIONES CONTEXTUALES: UN CENTRO CEREMONIAL EN LA EMERENCIANA}

La Emerenciana es un centro ceremonial Valdivia de importantes dimensiones (12,4 hectáreas de superficie total) ubicado encima de una playa colgada o playa fósil junto al río Buenavista en el sur de la provincia El Oro, Ecuador (Staller, 1994: 202-204). Las excavaciones en La Emerenciana permitieron descubrir porciones de una plataforma monticuliforme de tierra del Valdivia tardío con plataformas ovales de arcilla en su cima y muros artificiales de contención formando terrazas descendentes en los costados intactos norte y oeste de la estructura (Staller, 1994: 249-283). La investigación se concentró en el montículo artificial en el sector NO del sitio (Fig. 4). Las excavaciones incluyeron una serie de cortes (1-6) lo mismo que trincheras (A-D) y Perfil A, un corte de sedimentos expuestos de 29 metros de longitud (Fig. 5). Un total de seis capas estratigráficas con una interfase bien definida fueron distinguidas por el color y la textura(Fig.6, Tab.5). Éstas incluyeron dos pisos de ocupación Valdivia correspondientes a los Estratos 3 y 5. Una descripción y resumen breves de las diferentes capas estratigráficas es dada en la figura 6 y la tabla 5. Las excavaciones se hicieron en capas estratigráficas naturales, y se centraron en la identificación de los rasgos arqueológicos. Un total de 139 rasgos fueron identificados en los Estratos 5 y 3 incluyendo moldes de postes, pozos, rasgos asociados con la construcción del montículo artificial, diferentes ofrendas rituales y cuatro entierros Valdivia VII-VIII, tres de los cuales fueron identificados en la N-S Trinchera D (Staller, 1994: figure 22, tabla 18). Esta trinchera mide 12 metros de longitud; es cortada a través del centro de la cima del montículo.

La fase de ocupación La Valdivia VII-VIII en el sitio de La Emerenciana fue identificada en base a la evidencia estratigráfica, al diagnóstico cerámico, a varios fechados radiocarbónicos y a fechas por AMS. La ocupación Valdivia IV-VI asociada al Estrato 3 fue documentada por diagnóstico cerámico, evidencia estratigráfico y varios rasgos arqueológicos (Tab. 5). Las capas de ocupación, (Estratos 5 y 3) estaban separadas por una capa estéril de arena blanca (Estrato 4), que corresponde a la formación de dunas que representan el abandono breve del sitio. Las excavaciones en La Emerenciana comprendieron la documentación de los detalles arquitectónicos y rasgos arqueológicos asociados con un montículo ceremonial Valdivia en el sector NO del sitio (Staller, 1994: 249-283). Siete de las diez muestras de tiestos provienen de la capa de ocupación Valdivia VII-VIII (Estrato 5) y corresponden a contextos ceremoniales y a depósitos funerarios o rasgos que representan los restos materiales de ofrendas rituales. Las tres muestras de tiestos que poseen una evidencia clara de ópalofitolitos de maíz están asociadas con la ocupación de la Fase VII-VIII (Estrato 5).

\section{LOS ENTIERROS VALDIVIA VII-VIII EN LA EMERENCIANA}

Cuatro entierros Valdivia VII-VIII fueron encontrados en el sitio de La Emerenciana (Staller, 1994: 304-312) y todos están asociados estratigráficamente al Estrato 5 (piso 2), la capa de cenizas grises (Tab. 5). Cuando se dieron a conocer, representaban los únicos esqueletos Valdivia de esta secuencia cultural tardía. El entierro 1 estaba parcialmente perturbado y el cráneo ausente, mientras que el entierro 


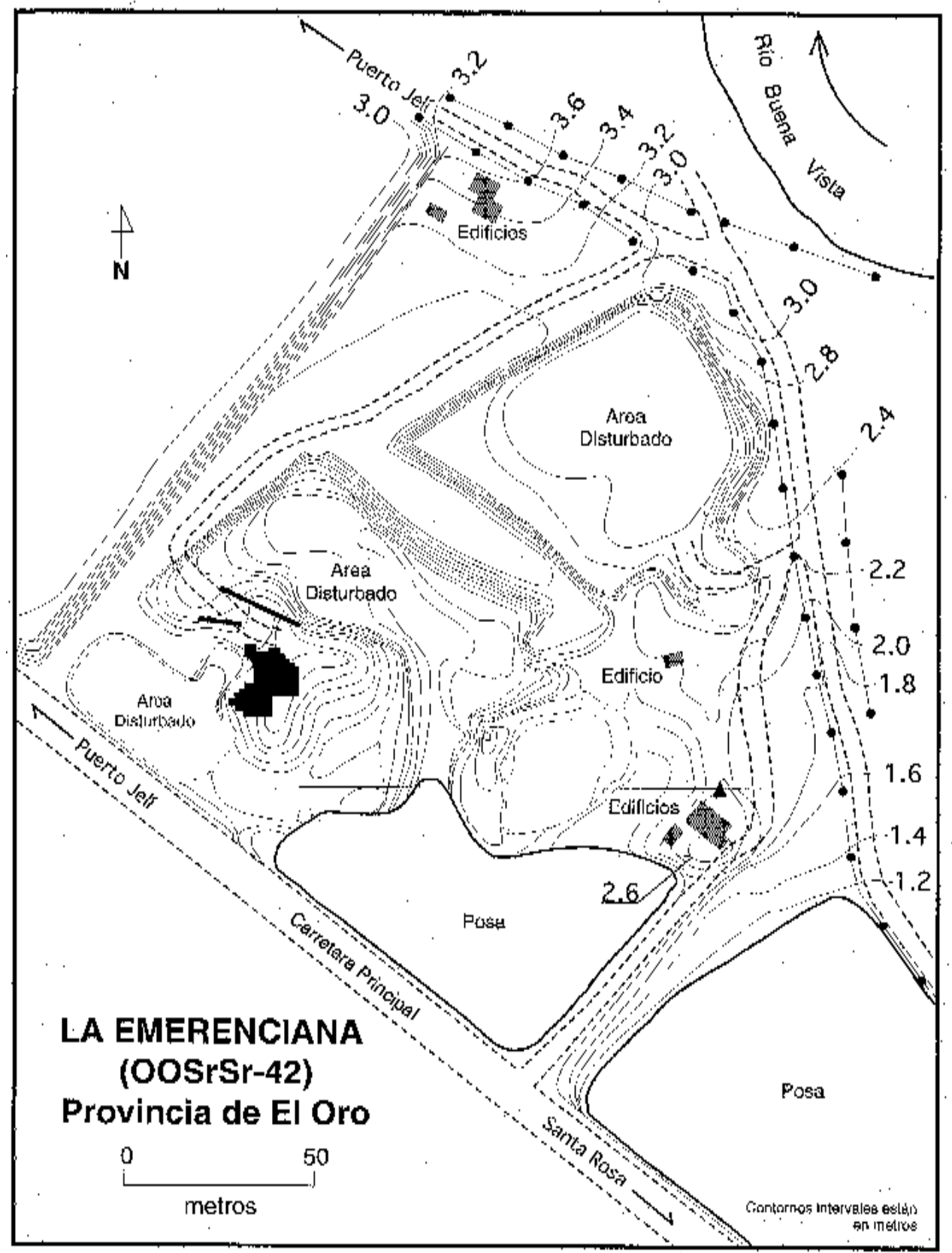

Fig. 4 - Mapa topográfico de La Emerenciana donde se señala el área excavada en el sector N.O. El montículo en la porción SE del sitio también representa una formación monticuliforme artificial abajo de construcciones modernas. 


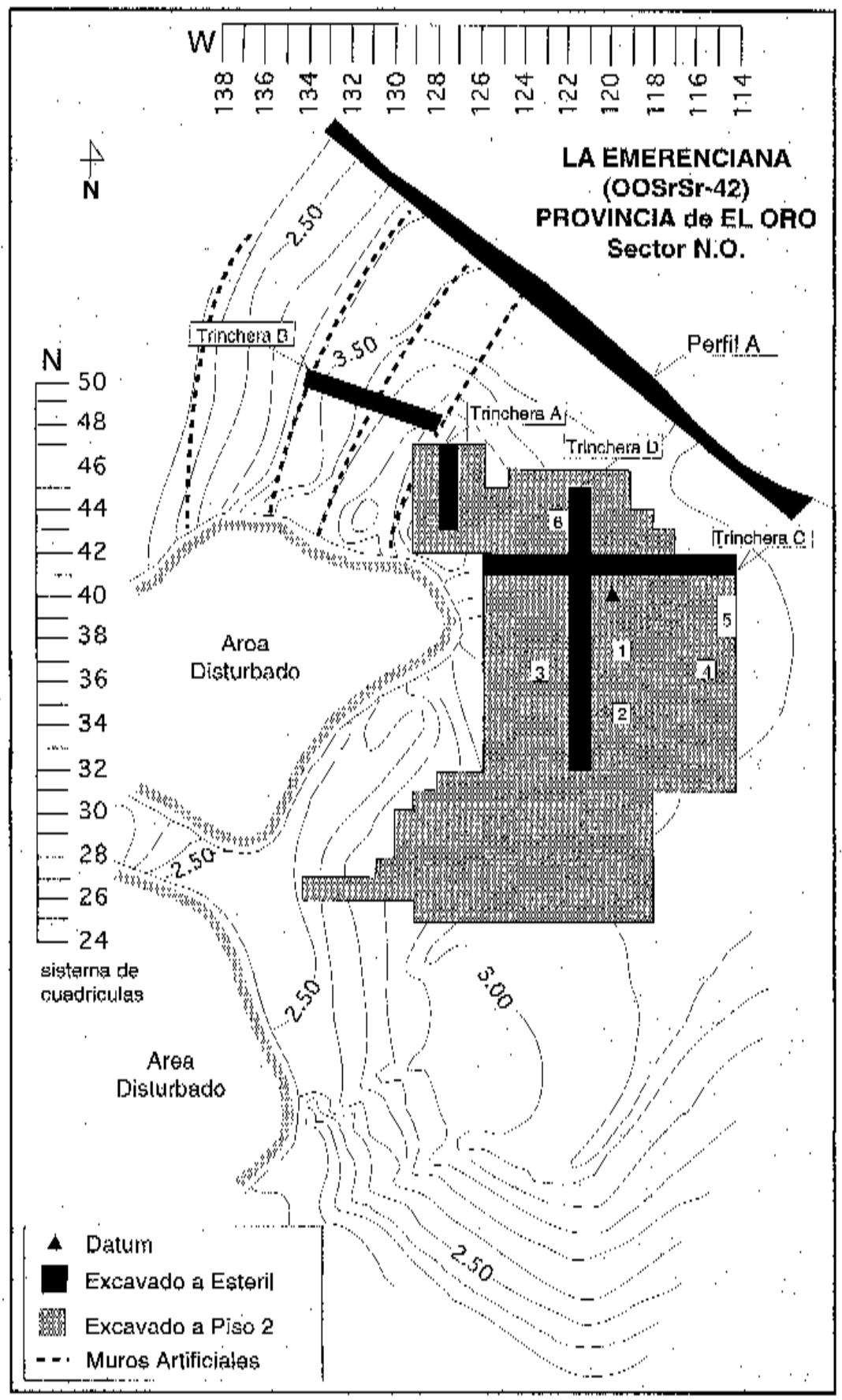

Fig. 5 - Área excavada en la porción N.O. del sitio. Se observan los distintos cortes (1-6), trincheras (A-D) y el perfil A. 


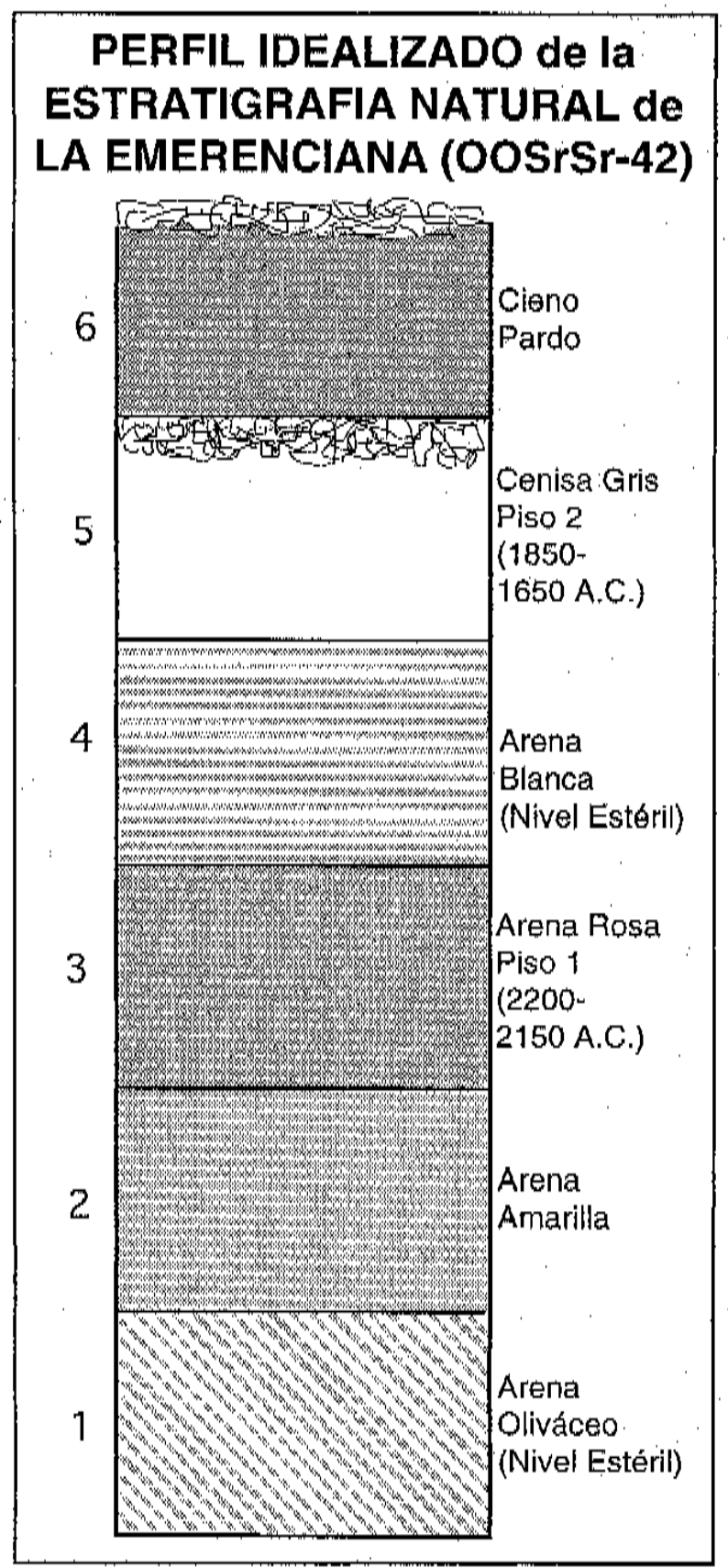

Fig. 6 - Perfil idealizado de la estratigrafía natural de La Emerenciana (para una descripción de las diferentes capas estratigráficas, véase la Tab. 5).

Nota: Las líneas encima de los estratos 5 y 6 representan concentraciones de concha. 
Tab. 5 - Principales capas estratigráficas de La Emerenciana (OO SrSr-42).

\begin{tabular}{|c|c|c|c|c|}
\hline Estra. & Profund. & Horiz. & Color & Descripción \\
\hline 6 & $0-55 \mathrm{~cm}$ & A & $10 y r 5 / 3$ & $\begin{array}{l}\text { Franco fino limoso pardo, flojamente consolidado } \\
\text { en los niveles superiores, más denso en los } \\
\text { inferiores, con evidencia de bioturbación (depósito } \\
\text { fluvial). }\end{array}$ \\
\hline 5 & $15-93 \mathrm{~cm}$. & B & $\begin{array}{l}10 y r \text { 6/1 } \\
-10 y r 5 / 1\end{array}$ & $\begin{array}{l}\text { Suelo franco gris ceniciento flojamente compac- } \\
\text { tado, textura, muy finas inclusiones cuarcíticas } \\
\text { con la consistencia del talco, y restos } \\
\text { artefactuales y de valvas en los niveles superiores } \\
\text { del estrato (piso de habitación 2) } \\
\text { (etnoestratigráfico). }\end{array}$ \\
\hline 4 & $36-92 \mathrm{~cm}$. & $\mathrm{C}$ & $10 y r 8 / 3$ & $\begin{array}{l}\text { Arena blanca de duna, finamente texturada, floja- } \\
\text { mente consolidada con inclusiones decarbonato } \\
\text { de calcio en los niveles superiores (depósito eólico). }\end{array}$ \\
\hline 3 & $78-145 \mathrm{~cm}$ & Bwn & $7,5 \mathrm{yr} 6 / 4$ & $\begin{array}{l}\text { Arena cuarzosa rosa finamente texturada, bien } \\
\text { consolidada, libre de inclusiones (piso habitación } \\
\text { 1) (etnoestratigráfico). }\end{array}$ \\
\hline 2 & $64-134 \mathrm{~cm}$ & Bwk & $\begin{array}{l}2,5 \text { y } 8 / 6 \\
-2,5 y 8 / 8\end{array}$ & $\begin{array}{l}\text { Arena amarilla finamente texturada, con } \\
\text { inclusiones de carbonato de calcio, inclusiones de } \\
\text { pequeños guijarros }(3 \mathrm{~mm}-1 \mathrm{~cm}) \text { (depósito eólico). }\end{array}$ \\
\hline 1 & 97- cm. & $\mathrm{C}$ & $\begin{array}{l}5 y 8 / 2 \\
-5 y 8 / 4\end{array}$ & $\begin{array}{l}\text { Arena blanco olivácea, texturada, flojamente } \\
\text { consolidada, finamente texturada, moderamente } \\
\text { compactada con pequeños guijarros }(3 \mathrm{~mm}-2 \mathrm{~cm}) \\
\text { costeros e inclusiones de carbonato de calcio) } \\
\text { (depósito fluvial). }\end{array}$ \\
\hline
\end{tabular}

Nota: Los colores del suelo están clasificados en base a la Munsell Soil Color Chart (1975). Las profundidades son bajo datum y se indican como niveles mínimos y máximos, los cuales obviamente variaron según las diferentes áreas de las excavaciones (según Staller, 1994: tabla 14).

3 era un subadulto articulado en el cual no se encontraron fitolitos en el tártaro. Pudimos solamente identificar fitolitos de tártaro dental en los entierros 2 y 4.

\section{ANÁLISIS DE TIESTOS VALDIVIA SELECCIONADOS Y DETÁRTARO DENTAL DE LA EMERENCIANA}

Las muestras de residuos alimenticios y tártaro dental fueron sumergidas en agua destilada y Alconox durante 12 horas para remover cualquier residuo de fitolitos que pudiera estar adherido. Después de un enjuague con agua destilada, las muestras de residuos fueron colocadas en ácido nítrico calentado durante un tiempo mínimo de 12 horas para disolver la porción orgánica. 
Las muestras de tártaro dental fueron tratadas primeramente con ácido hidroclorhídrico para separar los carbonatos y luego enjuagadas repetidamente con agua destilada y centrifugadas por espacio de 10 minutos a $3000 \mathrm{rpm}$. Después de repetir esta técnica cinco veces, las muestras fueron enjuagadas en alcohol dos veces y diez gotas de cada muestra fueron pipeteadas en portaobjetos y montadas en un medio permanente. Los fitolitos fueron contados usando un microscopio electrónico Leica Laborlux y fotografiadas con una impresora Video Color Sony.

\section{MATERIALES Y MÉTODOS: ANÁLISIS DE FITOLITOS}

Se examinaron diez tiestos Valdivia con residuos de alimentos carbonizados de las colecciones excavadas de La Emerenciana. Los residuos alimenticios fueron removidos de los tiestos raspando primeramente la capa en contacto con el suelo, y extrayendo luego el residuo remanente. Cada una de las muestras está compuesta por menos de 0,1 gramo de residuo alimenticio. La lista de procedencia de las muestras de residuos alimenticios y tártaro dental son presentadas en la Tab. 6.

Tab. 6 - Procedencias de las muestras de residuos de alimentos del sitio La Emerenciana (OOSrSr-42).

\author{
A. $\mathrm{N}^{\circ}$ de catálogo. 5480 \\ Procedencia: N37 W122 trinchera D \\ Capa: 5 \\ Rasgo: 1 (plataforma sur)
}

C. $\mathbf{N}^{\circ}$ de catálogo. 5623

Procedencia: N41 W122 trinchera D

Capa: 5

Rasgo: 65 (ofrenda en fosa con jarra invertida)

E. $\mathbf{N}^{\circ}$ de catálogo. 5618

Procedencia: N41 W122 trinchera D

Capa: 3

Rasgo: 137 (fosa)

G. $\mathbf{N}^{\circ}$ de catálogo. 5546

Procedencia: N44 W122 trinchera D

Capa: 5

Rasgo: 114 (fosa de ofrendas

estucada con arcilla)

I. $\mathbf{N}^{\circ}$ de catálogo. 4135

Procedencia: N40 W116 corte 5

Capa: 5

Rasgo: 56 (ofrenda monticular de valvas, restos faunísticos y cerámica partida)
B. $\mathbf{N}^{\circ}$ de catálogo. 5485

Procedencia: N38 W122 trinchera D

Capa: 5

Rasgo: 70 (ofrenda de una olla parada rota llena con restos de valvas, faunísticos y orgánicos)

D. $\mathbf{N}^{\circ}$ de catálogo. 5430

Procedencia: N41 W126 trinchera C Capa: 3

Rasgo: 47 (impronta de poste con olla rota en la base)

F. $\mathbf{N}^{\circ}$ de catálogo. 4268

Procedencia: N37 W124 corte 3

Capa: 6

Tiesto de la superficie del Estrato 5

H. $\mathbf{N}^{\circ}$ de catálogo. 5534

Procedencia: N34 W122 trinchera D

Capa: 5

Rasgo: 90 (fosa de ofrendas estucada con arcilla)

J. $\mathbf{N}^{\circ}$ de catálogo. 5429

Procedencia: N42 W123 corte 6

Capa: 5

Rasgo: 117 (fosa funeraria, Entierro

4) 


\section{ANÁLISIS DE ISÓTOPOS DE CARBONO}

Se removió la capa de residuos alimenticios que había estado en contacto con el suelo en cinco tiestos seleccionados. Los residuos alimenticios restantes fueron raspados sobre papel de cromatografía, colocados en contenedores de vidrio y luego enviados a Beta Analytic para su ulterior procesamiento.

\section{RESULTADOS ANALÍTICOS: ANÁLISIS DE FITOLITOS}

Nuestros resultados indican que tres de las siete muestras de tiestos del Estrato 5 de La Emerenciana contienen un conjunto de fitolitos en los que predominan los del tipo rondels. Los fitolitos pueden ser producidos por una variedad de gramíneas y la inflorescencia de algunas de ellas producen conjuntos de rondeles. Las muestras del Estrato 3 produjeron pocos fitolitos, reflejando una ausencia de plantas productoras de sílice. Nuestros resultados son presentados en la Tab. 7 y un cuadro gráfico comparativo es presentado en la Fig. 7. Ambas muestras de tártaro dental contenían ópalofitolitos, el entierro 2 presentaba una cantidad significativamente mayor, pero era también una muestra más grande que la obtenida del entierro 4 (Tab. 8).

Tab. 7 - Cuerpos silíceos de gramínea recuperados de muestras seleccionadas de residuos de alimentos del sitio de La Emerenciana (OOSrSr-42).

\begin{tabular}{|c|c|c|c|c|c|}
\hline & $\begin{array}{c}\text { A } \\
\text { Estrato } 5 \\
\text { Rasgo } 1\end{array}$ & $\begin{array}{c}\text { B } \\
\text { Estrato } 5 \\
\text { Rasgo } 70\end{array}$ & $\begin{array}{c}\text { C } \\
\text { Estrato } 5 \\
\text { Rasgo } 65\end{array}$ & $\begin{array}{c}\text { D } \\
\text { Estrato } 3 \\
\text { Rasgo } 47\end{array}$ & $\begin{array}{c}\text { E } \\
\text { Estrato } 3 \\
\text { Rasgo } 137\end{array}$ \\
\hline Rectángulo & 2 & 3 & 2 & 0 & 3 \\
\hline Cruz & 2 & 1 & 0 & 0 & 2 \\
\hline Sinuoso & 1 & 1 & 1 & 0 & 0 \\
\hline Bilobulado & 3 & 3 & 2 & 1 & 0 \\
\hline Rondel, E.M.* & 3 & 11 & 7 & 0 & 3 \\
\hline Rondel, I.M.* & 35 & 33 & 34 & 0 & 5 \\
\hline Rondel, E.E.* & 7 & 9 & 7 & 0 & 0 \\
\hline Rondel I.E.* & 55 & 47 & 52 & 1 & 3 \\
\hline Montura & 0 & 1 & 0 & 2 & 1 \\
\hline Total & 108 & 108 & 112 & 4 & 17 \\
\hline
\end{tabular}

* Los fitolitos Rondel han sido clasificados según si la faceta delgada es entera o indentada (E. o I.) y si la faceta espesa es entera o en muesca (E. o M.).

Además de los cuerpos de sílice en gramíneas, otros ópalofitolitos se recuperaron, incluyendo esferas espinosas, que pueden estar indicando la presencia tanto de palmera como de 'raíz de flecha' (Maranta arundinacea) (Pearsall, 1992a:195; 1994a) y están presentes en muestras de los Estratos 5 y 3 . Un ejemplo está ilustrado en la Fig. 8. 


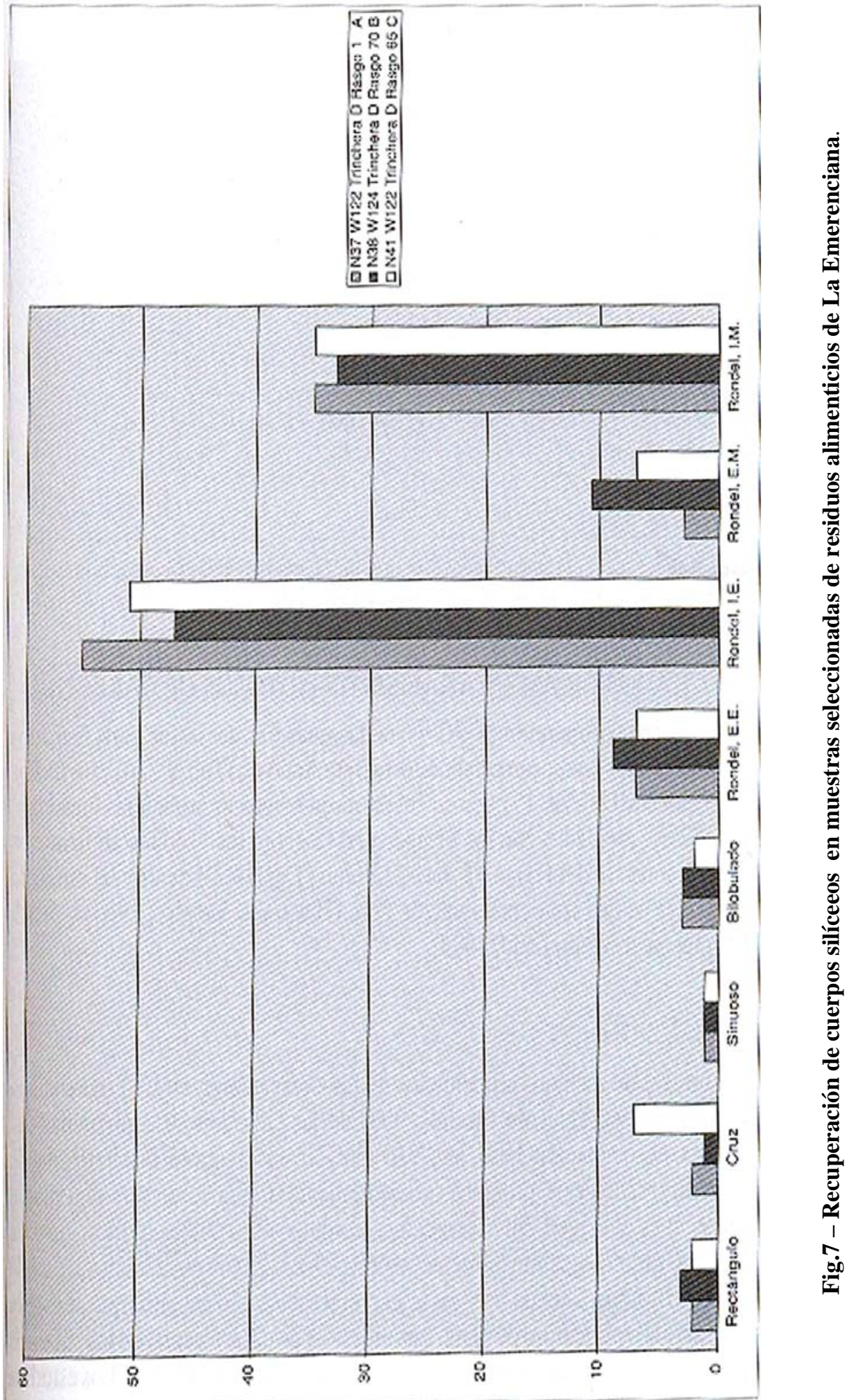


Tab. 8 - Cuerpos silíceos recuperados de muestras de tártaro seleccionadas del sitio de La Emerenciana (OOSrSr-42).

\begin{tabular}{|lcc|}
\hline & Entierro 2 & Entierro 4 \\
Rectángulo & 0 & 0 \\
Cruz & 0 & 0 \\
Sinuoso & 1 & 0 \\
Bilobulado & 0 & 0 \\
Rondel, E.M.* & 3 & 0 \\
Rondel, I.M.* & 6 & 3 \\
Rondel, E.E.* & 3 & 2 \\
Rondel I.E.* & 4 & 1 \\
Montura & 0 & 0 \\
Inclinados Rondels & 41 & 6 \\
Total & 58 & 12 \\
\hline
\end{tabular}

* Los fitolitos Rondel han sido clasificados según si la faceta delgada es entera o indentada (E. o I.) y si la faceta espesa es entera o en muesca (E. o M.).

\section{FECHADO POR AMS Y ANÁLISIS DE ISÓTOPOS DE CARBONO}

$\mathrm{El}$ análisis de la proporción de ${ }^{13} \mathrm{C} /{ }^{12} \mathrm{C}$ de las muestras de tiestos y los fechados de AMS fueron llevados a cabo por el laboratorio Beta Analytic (Tab. 9y 10) y las fechas radiocarbones por SMU (Tab. 11). No se encontraron rasgos ni problemas inusuales. Las tasas de ${ }^{13} \mathrm{C} /{ }^{12} \mathrm{C}$ asociadas con los fechados AMS sobre los residuos alimenticios contenidos en la muestra del Rasgo 65 fue -21,9o/oo (Tab. 10, B) dentro del rango de residuos alimenticios que contienen maíz de Perú (Hastorf \& DeNiro, 1985) y North Dakota (Thompson \& Mulholland, 1994).

\section{DISCUSIÓN}

Las muestras de residuos alimenticios de las excavaciones en La Emerenciana contienen conjuntos variables de fitolitos. Tres de las siete muestras obtenidas del Estrato 5 (capa de ocupación Fase VII-VIII) indicó un conjunto que reflejaba la presencia de inflorescencia de gramínea suficientemente similar como para sugerir que la misma especie es el origen. Su asociación a residuos alimenticios indica el uso de inflorescencia de gramínea como alimento, en este caso, el maíz es la fuente lógica de los conjuntos de fitolitos. Dichos fitolitos eran compatibles con aquellos que se espera encontrar en marlos o pericarpio de maíz. Las muestras de tártaro dental de los entierros 2 y 4 contienen un conjunto de formas rondel compatibles con las de maíz. Los estudios de isótopos de los esqueletos Valdivia de La Emerenciana realizados por Tykot (1996) sugieren que plantas $\mathrm{C} 4$ tales como el maíz están claramente presentes en la dieta de los ocupantes prehistóricos asociados con la ocupación del Estrato 5. Las dos muestras de 


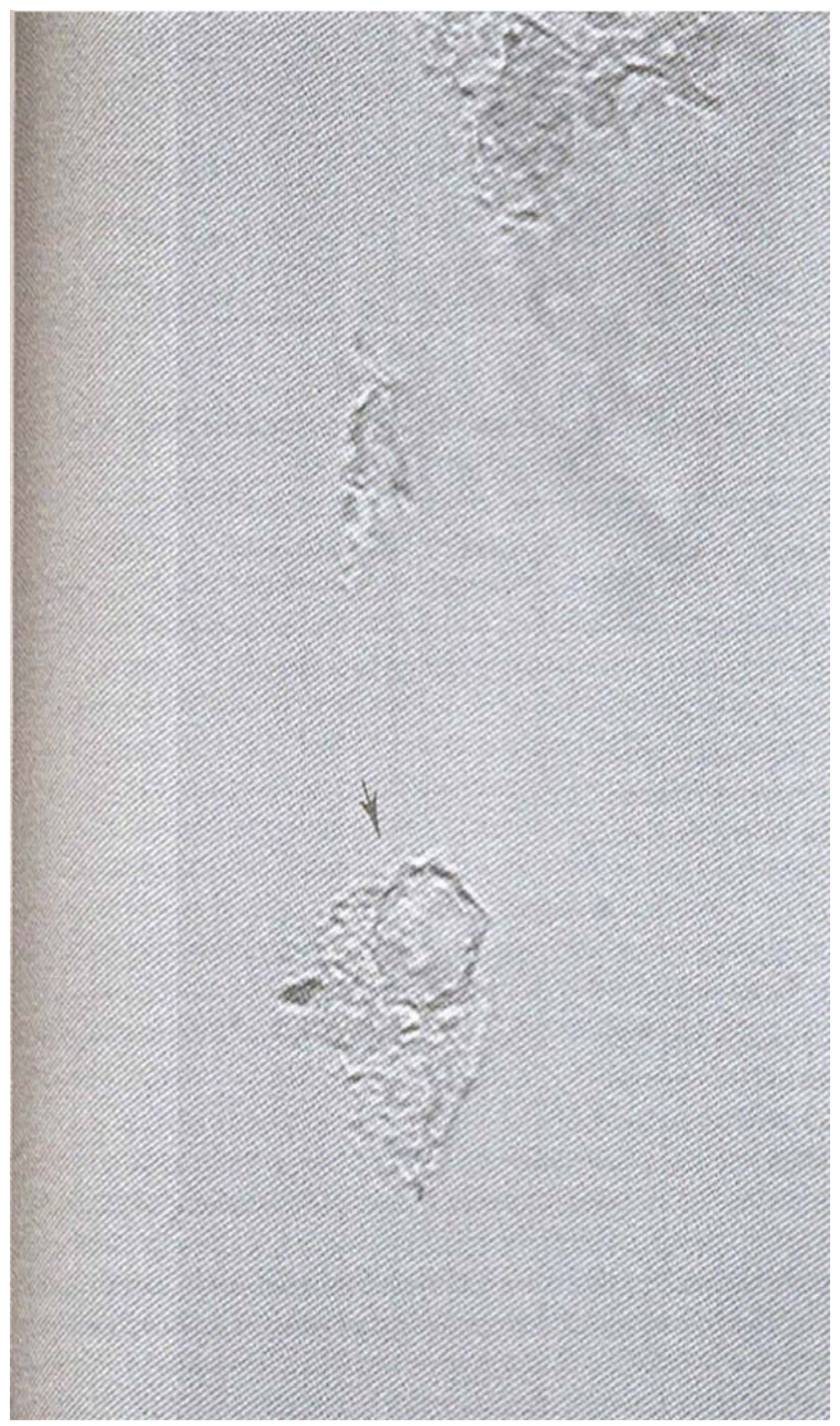


Tab. 9 - Proporciones ${ }^{13} \mathrm{C} /{ }^{12} \mathrm{C}$ de las muestras seleccionadas de residuos carbonosos de la cerámica Valdivia del sitio de La Emerenciana (OOSrSr-42).

\begin{tabular}{|ccccc|}
\hline $\mathbf{N}^{\circ}$ de catálogo & Beta $\mathbf{N}^{\circ}$ & Capa & Rasgo & Proporción ${ }^{\mathbf{1 3}} \mathbf{C}^{{ }^{\mathbf{1 2}} \mathbf{C}}$ \\
\hline 5430 & 84310 & 3 & 47 & $-26,1$ \\
5485 & 84311 & 5 & 70 & $-26,2$ \\
5618 & 84312 & 3 & 137 & $-26,7$ \\
5623 & 84313 & 5 & 65 & $-23,8$ \\
5480 & 84314 & 5 & 1 & $-26,2$ \\
\hline
\end{tabular}

Tab. 10 - Fechados de acelerador de masa espectrometría (AMS) de La Emerenciana (OOSrSr-42).

\begin{tabular}{|c|c|c|c|}
\hline Datos de la muestra & $\begin{array}{c}\text { Edad }{ }^{14} \mathrm{C} \\
\text { Medida }\end{array}$ & $\begin{array}{c}\text { Proporción } \\
{ }^{13} \mathrm{C} /{ }^{12} \mathrm{C}\end{array}$ & $\begin{array}{c}\text { Edad }{ }^{{ }^{14} \mathrm{C}} \\
\text { convencional }\end{array}$ \\
\hline $\begin{array}{l}\text { a) Beta-125106 } \\
\text { Muestra A\#: } 5480 \\
\text { Análisis: Standard AMS } \\
\text { Material/Pretratamiento: } \\
\text { (material orgánico/lavados } \\
\text { ácidos) }\end{array}$ & $3720 \pm 40$ A.C. & $-25,8 \%$ & $\begin{array}{c}\text { 3700 } \pm 40 \text { A.C. } \\
\text { (calibrada } \\
2310 \text { A.C.) }\end{array}$ \\
\hline $\begin{array}{l}\text { b) Beta-125107 } \\
\text { Muestra B\#: } 5623 \\
\text { Análisis: Standard AMS } \\
\text { Material/Pretratamiento: } \\
\text { (material orgánico/lavados } \\
\text { ácidos) }\end{array}$ & $3810 \pm 50$ A.C. & $-21,9 \%$ & $\begin{array}{c}\text { 3860 } \pm 50 \text { A.C. } \\
\text { (calibrada } \\
2310 \text { A.C.) }\end{array}$ \\
\hline
\end{tabular}

tiestos obtenidas del Estrato 3 y una del Estrato 6 rindieron menor cantidad de sílice sugeriendo que el material de inflorescencia de gramínea no estaba incorporado en los residuos.

La presencia de fitolitos puede confirmar la existencia de maíz en una muestra, el porcentaje de la muestra formado por la planta no puede ser determinado, excepto por el análisis detallado de las tasas $d e^{13} \mathrm{C} /{ }^{12} \mathrm{C}$ de especies vegetales que puedan formar parte de los restos de residuos alimenticios. Aún sin este análisis detallado, se pueden intentar algunas comparaciones. Por ejemplo, en tanto parece haber una tendencia general hacia números menos negativos en las tasas $\mathrm{de}^{13} \mathrm{C} /{ }^{12} \mathrm{C}$, la diferencia no es muy grande. Sólo una única muestra, recuperada de una vasija restringida invertida en el Rasgo 65 puede 
Tab. 11 - Fechados radiocarbónicos de la Emerenciana (OOSrSr-42).

\begin{tabular}{|c|c|c|}
\hline $\begin{array}{l}{ }^{14} \mathrm{C} \mathrm{N}^{\circ} \text { de laboratorio } \\
\text { (material) }\end{array}$ & $\begin{array}{l}\text { Medición } \\
{ }^{14} \mathrm{C} \text { Edad }\end{array}$ & $\begin{array}{c}\text { Calibrado } \\
2 \text { Sigma, } 95 \%\end{array}$ \\
\hline $\begin{array}{l}{ }^{14} \mathrm{C} \mathrm{N}^{\circ} \mathrm{SMU}-2225 \\
\text { (carbón) }\end{array}$ & 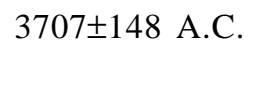 & $2203 \pm 215$ A.C. \\
\hline $\begin{array}{l}{ }^{14} \mathrm{C} \mathrm{N}^{\circ} \mathrm{SMU}-2226 \\
\text { (carbón) }\end{array}$ & $3400 \pm 220$ A.C. & $1690 \pm 310$ A.C. \\
\hline $\begin{array}{l}{ }^{14} \mathrm{C} \mathrm{N}^{\circ} \mathrm{SMU}-2241 \\
\text { (carbón) }\end{array}$ & 3361士246 A.C. & 1679 \pm 300 A.C. \\
\hline $\begin{array}{l}{ }^{14} \mathrm{C} \mathrm{N}^{\circ} \mathrm{SMU}-2563 \\
\text { (carbón) }\end{array}$ & 3775 \pm 165 A.C. & $1846 \pm 165$ A.C. \\
\hline
\end{tabular}

decirse que contenía evidencia más fuerte del uso del maíz (Tab. 8, B). Es importante notar que la técnica de procesamiento utilizada por Beta Analytic emplea un lavado ácido que impide la inclusión de aquel material orgánico encerrado en los fitolitos contenidos en los residuos alimenticios (Tab. 9). La inclusión de material orgánico de los fitolitos requiere técnicas de procesamiento especiales (Mulholland \& Prior, 1993). La existencia de maíz puede ser confirmada en tres de las siete muestras de residuos alimenticios presentes en el Estrato 5 de La Emerenciana. No se puede confirmar su presencia en residuos alimenticios del Estrato 3. Sin embargo, esto no demuestra una ausencia de maíz en la ocupación Valdivia representada en el Estrato 3. Más aún, el tamaño de la muestra es demasiado pequeño (dos muestras) como para habilitarnos para negar la presencia del maíz en el estrato. En honor a la verdad, debemos manifestar que hasta el presente no hay evidencia directa de la existencia de plantas C4 en el Estrato 3 de La Emerenciana.

Las muestras de residuos alimenticios del Estrato 5 de La Emerenciana son derivadas exclusivamente de los contextos ceremoniales. Su identificación con ofrendas rituales y una vasija restringida Valdivia VIII, sugiere que la planta fue más probablemente consumida como intoxicante fermentado (Cutler \& Cárdenas, 1947; Moore, 1989). Las asociaciones contextuales sugieren que el maíz ha sido utilizado como planta sagrada. Tal evidencia contextual da apoyo a una interpretación similar hecha por Burger y van der Merwe (1990) que implica la dispersión temprana de esta planta en el occidente de Sudamérica. Esta evidencia contextual de La Emerenciana sugiere que si el maíz era consumido, probablemente lo fuera en forma líquida como un alcoholizante fermentado o chicha durante los rituales ceremoniales (ver Burger \& van der Merwe, 1990; Cutler \& Cárdenas, 1947; Lathrap et al., 1975; Stahl, 1984). La evidencia directa que sostiene tal conclusión viene de la presencia de conjuntos de fitolitos de maíz hallados en los tiestos basales del Rasgo 65, una vasija evertida con un fechado asociado por AMS de $3860 \pm 50$ A.P. (Tab. 8, B). Esta fecha de AMS es de mismo rasgo y contexto de fecha 
radiocarbónica de SMU-2563 donde obtenemos una fecha de 3775 \pm 165 A.P. Estos datos cronológicos indican una introducción en fechas calibradas entre 2240-2201 A.C. (para la fecha AMS) y entre 2459-1922 A.C. para la fecha radiocarbónica. Con estos datos podemos determinar con más confianza una introducción del maíz en La Emerenciana entre ca., 2200 y 1950 A.C. Esto es en el mismo tiempo que la proliferación de varias clases de botellas simples, asas estribos, y jarras en el complejo cerámico de la Fase Jelí de La Emerenciana (Staller, 1992-1993: figuras 9a, 9b, 10a-c, 11a, b; 1994 : 352-390, figuras 46-53; 2001) daría sostén a la idea de la utilización amplia del maíz en forma de chicha. A partir de esta evidencia nos se descarta necesariamente la utilización del maíz en tanto planta con valor económico durante las fases VII-VIII, sino que, conforme con la evidencia presentada en este análisis, se considera que también fue una planta ritual con significado ceremonial para la cultura Valdivia. De este modo, el consumo del maíz como un intoxicante ritual podría ayudar a explicar por qué se dispersó tan rápidamente por todo el mundo andino en una variedad de contextos culturales luego de su introducción inicial en la costa ecuatoriana.

El análisis de ópalofitolitos pudo proporcionar datos para detectar la presencia de maíz en las vasijas cerámicas de La Emerenciana, sitio datado en las épocas finales (Fases VII-VIII) del Período Formativo Temprano de la costa del Ecuador (Hill, 19721974: 21). Tanto la palmera como la raíz de flecha están también presentes. Ambos eran componentes de la dieta Valdivia más temprana en otras regiones de la costa del Ecuador (Pearsall, 1992a: 188; 1992b: figura 9.4; 1994a).

Nuestro enfoque contextual y metodológico responde a interrogantes surgidos a partir de la evidencia previa de una introducción temprana del maíz en Sudamérica (Bruhns, 1994; Fritz, 1994a; 1994b; Smith, 1998). Sobre la base de la evidencia existente es posible concluir que el maíz estaba presente durante las fases VII y VIII (Estrato 5) de la fase Valdivia en La Emerenciana (ver Fig. 6). La existencia e importancia del maíz en la subsistencia Valdivia durante las porciones más tempranas de la secuencia cultural carece de sustento (ver por ej., Pearsall, 1978; 1993; Pearsall \& Piperno, 1990; Zevallos, 1971; Zevallos et al., 1977).

La evidencia analítica de estudios isótopicos previos de colágeno óseo ecuatoriano prehistórico también sostiene nuestras conclusiones puesto que ninguna muestra de las más tempranas Fases I-IV indican que las gramíneas C4 hayan sido un componente de menos importancia en la dieta Valdivia (van der Merwe et al., 1993: tabla 2; Tykot, 1996). El colágeno óseo de los entierros 2 y 4 de La Emerenciana indicaron un incremento en el consumo de gramíneas C4 (Tykot, 1996). Los datos isotópicos implican que el incremento en el consumo de gramíneas C4 durante los tiempos Valdivia VII-VIII es compatible con la evidencia química clara del consumo de maíz a partir de los esqueletos de la Fase Machalilla en su muestra (van der Merwe et al., 1993: tabla 2; Tykot, 1996). Nuestra conclusión además está sostenida por un análisis de los patrones de asentamiento Valdivia que indican un cambio en el interior de la región durante la porción final (Fase VI-VIII) de la secuencia cultural (Staller, 1994: figura 5) y un incremento dramático de la población correspondiente a la Fase de ocupación Chorrera del Formativo Tardío. 
En suma, el maíz está presente durante las Fases VII-VIII de la ocupación Valdivia de La Emerenciana. La presencia de la especie en las fases más tempranas permanece carente de sustento. Hasta que las cuestiones contextuales que surgen a partir de los análisis de sedimentos se resuelvan, nuestras conclusiones se resumen como sigue: la evidencia arqueológica de los residuos alimenticios encontrados en cerámica Valdivia y los datos isotópicos de los dos entierros de La Emerenciana, así como también el análisis de colágeno óseo de una muestra grande de esqueletos prehistóricos ecuatorianos indican que el maíz se incorpora a la dieta Valdivia no antes de alrededor de 2200 a 1950 A.C. La afirmación de que el consumo de la planta de maíz C4 fue un rasgo crítico de la subsistencia Valdivia antes de las Fases VII and VIII no está sustentada por la evidencia presentada en este análisis ni por los estudios isotópicos de individuos prehispánicos del Ecuador de van der Merwe et al. (1993). Sobre bases contextuales, su introducción en la dieta es interpretada como una planta sagrada inicialmente consumida como un intoxicante fermentado en la forma de chicha.

Los métodos de investigación y los análisis de fitolitos presentados en este estudio no deberían limitarse en los estudios futuros estrictamente a los residuos en vasijas cerámicas, particularmente en la costa del Ecuador, una región donde los macrofósiles de plantas son notoriamente raros. El muestreo de fitolitos será necesario en estudios futuros para obtener una comprensión más clara de paleodietas y actividades de procesamiento, así como de la subsistencia Valdivia y los orígenes de la agricultura. Nuestra metodología de investigación aguarda a más paleobotánicos y arqueólogos especializados en otras regiones de Sudamérica.

\section{Agradecimientos}

Las excavaciones de gran escala en el sitio de La Emerenciana fueron posibles gracias a los fondos aportados por la Fulbright Doctoral Dissertation Research Abroad Grant otorgada a John E. Staller y fueron llevados a cabo bajo el auspicio del Museo Antropológico del Banco Central del Ecuador, Guayaquil, Ecuador. La extracción de fitolitos a partir de residuos alimenticios fue realizada en las instalaciones del laboratorio cedido por el Profesor David Biesboer del Departamento de Biología Vegetal de la Universidad de Minnesota. El Dr. Paul Burkhouse supervisó y ayudó en el análisis de cromatografía de papel. Woodward-Clyde Consultants Inc., proporcionó fondos y el microscopio posibilitando el análisis de isótopos fue proporcionado por Beta Analytic. Deseamos extender nuestra gratitud sincera a Bruce D. Smith de la Smithsonian Institution por su amabilidad y generosidad al propocionar los fondos para procesar las dataciones de AMS a partir de las muestras de los residuos de carbón presentadas en este estudio y por su constante apoyo a esta investigación. Nuestro agradecimiento al Directorio del Institute for Minnesota Archaeology, por proporcionarnos los fondos necesarios para escribir partes de este artículo. También queremos expresar nuestra gratitud a los muchos revisores 
anónimos que cuidadosamente leyeron y mejoraron la comprensión de los primeros borradores de este manuscrito. Muchas gracias a mis colegas argentinos de la Universidad Nacional de Río Cuarto (UNRC), María A. Bordach (M.A.) y Osvaldo J. Mendonça (M.A.), por la traducción de este manuscrito al castellano.

\section{Referencias citadas}

BENZ, B. F., 1998 - On the origin, evolution, and dispersal of maize. In: Pacific Latin America in prehistory: The evolution of archaic and formative cultures (M. Blake, ed.): 25-38; Pullman: Washington State University Press.

BENZ, B. F., \& ILTIS, H. H., 1990 - Studies in archaeological maize. I. The "wild" maize from San Marcos Cave reexamined. American Antiquity, 55: 500-511.

BENZ, B. F., \& LONG, A., 2000 - Prehistoric maize evolution in Tehuacan Valley. Current Anthropology, 41(3): 459-465.

BIRD, R. M., 1978 - Archaeological maize from Peru. Maize Genetics Cooperative Newsletter, 52: $90-92$.

BIRD, R. M., 1979 - The Evolution of Maize: A New Model for the Early Stages. Maize Genetics Cooperation News Letter, 53: 53-54

BIRD, R. M., 1984 - South American Maize in Central America? In: Pre-Columbian Plant Migration (D. Stone, edit.): 39-65. Harvard University, Peabody Museum of Archaeology and Ethnology Papers, Vol. 76. Papers presented at the 44th International Congress of Americanist, Manchester, England.

BIRD, R. M., 1990 - What are the chances of finding maize in Peru dating before 1000 B.C.? American Antiquity, 55(4): 828-840.

BIRD, R. M. \& BIRD, J. B., 1980 - Gallinazo maize from the Chicama Valley, Peru. American Antiquity, 45(3): 325-333.

BOZARTH, S., 1987 - Diagnostic Opal Phytoliths from Rinds of Selected Curcurbita Species. American Antiquity, 52(3): 607-615.

BONAVIA, D. \& GROBMAN, A., 1989a-Preceramic maize in the Central Andes: A necessary clarification. American Antiquity, 54(4): 836-840.

BONAVIA, D. \& GROBMAN, A., 1989b - Andean maize: It's origins and domestication. In: Foraging and Farming: The evolution of plant exploitation (D.R. Harris \& C.G. Hillman, eds.): 456-470; London: Uwin-Hyman.

BRUHNS, K. O., 1994 - Ancient South America, 424p.; Cambridge: Cambridge World Archaeology-Cambridge University Press.

BRAUN, D., 1983 - Pots as Tools. In: Archaeological Hammers and Theories (J. A. Moore \& A. S. Keegan, eds.): 153-181; N.Y.: Academic Press.

BRAUN, D., 1987 - Coevolution of Sedentism, Pottery Technology, and Horticulture in the Central Midwest 200 B.C. - A.D. 600. In: Emergent Horticultural Economies of the Eastern Woodlands (W.F. Keegan, edit.): 153-181; Center for Archaeological Investigations, Southern Illinois University. Occasional Paper $N^{\circ} 7$.

BURGER, R. L. \& MERWE van der, N. J., 1990 - Maize and the Origin of highland Chavín Civilization. American Anthropologist, 92(1): 85-95.

BUSH, M.B., PIPERNO, D. R. \& COLINVAUX, P. A., 1989 - A 6000 year history of Amazonian maize cultivation. Nature, 340(6231): 303-305.

CUTLER, H. C. \& CÁRDENAS, M., 1947 - Chicha, A Native South American Beer. Botanical Museum Leaflets, 13: 33-60; Harvard University.

DENIRO, M. J., 1987 - Stable Isotopy and Archaeology. American Scientist 75(2): 182-191. 
DOEBLEY, J. F. 1990 - Molecular Evidence and the Evolution of Maize. Economic Botany 44(3 Supplement): 6-27.

DOEBLEY, J. F., GOODMAN, M. M. \& STUBER, C. W., 1986 - Exceptional Genetic Divergence of Northern Flint Corn. American Journal of Botany, 73(1): 64-69.

DOEBLEY, J. F., STEC, A. \& HUBBARD, L., 1997 - The Evolution of Apical Dominance in Maize. Nature, 386: 485-488.

DOOLITTLE, W. E. \& FREDRICK, C. D., 1991 - Phytoliths as indicators of prehistoric maize (Zea mays subsp. mays, Poaceae) cultivation. Plant Systemics and Evolution, 177: 175184.

DORWEILER, J. \& DOEBLEY, J. F., 1997 - Developmental analysis of teosinte glume architecture 1: A key locus in the evolution of maize (Poaceae). American Journal of Botany, 84(10): 1313-1322.

DORWEILER, J., STEC, A., KERMICLE, J. \& DOEBLEY, J. F., 1993 - Teosinte Glume Architecture 1: A genetic locus controlling a key step in maize evolution. Science, 262: 233-235.

EDMAN, G. \& SODERBERG, E., 1929 - Auffindung von reiss in einer tonscherbe aus einer etwas funftqusenjahreugen Chinesischen siedlung. Bulletin of the Geological Society of China, 8: 363-365.

EVANS, Jr., C., \& MEGGERS, B. J., 1966 - Beginnings of food production in Ecuador. In: Actas y memorias del 36th Congreso Internacional de Americanistas, Volume 1: 201-207.

FRITZ, G., 1994a - Are the First American Farmers Getting Younger? Current Anthropology, 35(3): 305-309.

FRITZ, G., 1994b - Reply to Piperno. Current Anthropology, 35(3): 639-642.

HASTORF, C. A., 1994 - The changing approaches to maize research. In: History of Latin American Archaeology (A. Oyuela-Caydedo, edit.): 139-154; Avebury, Brookfield, U.S.A.

HASTORF, C. A. \& DENIRO, M. J., 1985 - Reconstruction of Prehistoric Plant Production and Cooking Practices by a new Isotopic Method. Nature, 315: 489-491.

HERON, C., EVERSHED, R. \& GOAD, L., 1991 - Effects of Migration of Soil Lipids on Organic Residues Associated with Buried Potsherds. Journal of Archaeological Science, 18: 641659.

HILL, B. D., 1972-1974 - A new chronology of the Valdivia ceramic complex from the coastal zone of Guayas Province, Ecuador. Nawpa Pacha, 10-12: 1-32; Berkeley.

KELLY, E. R., MARINO, A. B. \& DENIRO, M. J., 1991 - Stable isotope ratios of carbon in phytoliths as a quantitative method of monitoring vegetation and climate change. Quaternary Research, 35: 222-233.

LATHRAP, D. W., COLLIER, D. \& CHANDRA, H., 1975 - Ancient Ecuador Culture, Clay, and Creativity 3000-300 BC, 110p.; Chicago: Field Museum of Natural History.

LATHRAP, D. W., MARCOS, J. G. \& ZEIDLER, J. E., 1977 - Real Alto: An Ancient Ceremonial Center. Archaeology, 30(1): 2-13.

LETTS, J., EVANS, J., FUNG, M. \& HILLMAN, G. C., 1994 - A Chemical Method of Identifying Charred Plant Remains Using Infra-red Spectroscopy. In: Corn and Culture in the Prehistoric New World (S. Johannessen \& C. Hastorf, edit.): 64-89; Boulder: Westview Press.

MEGGERS, B. J. 1966 - Ecuador Ancient peoples and places series 49, 220p.; N.Y.: G. Daniel Praeger Publication N.Y.

MEGGERS, B. J., EVANS Jr., C. \& ESTRADA, E., 1965 - Early Formative of Coastal Ecuador: The Valdivia and Machalilla Phases, 243p.; Washington D.C. : Smithsonian Contributions in Anthropology, Volume 1.

METCALF, C. R., 1960 - Anatomy of the Monocotyledons. I. Graminae; London: Oxford University Press. 
MOORE, J. D., 1989 - Pre-Hispanic Beer in Coastal Peru: Technology and Social Context of Prehistoric Production. American Anthropologist, 91: 682-695.

MULHOLLAND, S. C., 1990 - Arunda donax phytolith assemblages. Phytolitherian, 6(2): 3-9.

MULHOLLAND, S. C. \& PRIOR, C., 1993 - AMS Radiocarbon Dating of Phytoliths. In: Current Research in Phytolith Analysis: Applications in Archaeology and Paleoecology, (D.M. Pearsall \& D.R. Piperno, edit.): 21-23; Philadelphia: University of Pennsylvania. MASCA Research Papers in Science and Archaeology.

MULHOLLAND, S. C. \& RAPP, G., 1992 - A Morphological Classification of Grass Silica Bodies. In: Phytolith Systematics: Emerging Issues (G. Rapp \& S. Mulholland, edit): 6589; N.Y. : Plenum Press. Advances in Archaeological and Museum Science, Volume 1.

PEARSALL, D. M., 1978 - Phytolith analyses of archaeological soils: Evidence of maize cultivation in Formative Ecuador. Science, 199: 177-178.

PEARSALL, D. M, 1982 - Phytolith analysis: The application of a new paleoethno-botanical technique in archaeology. American Anthropologist, 84: 862-871.

PEARSALL, D. M., 1987 - Evidence for prehistoric maize cultivation on raised fields at Peñon del Río, Guayas, Ecuador. In: Pre-Hispanic Agricultural Fields in the Andean Region (Part 2): 279-296. BAR International Series 359.

PEARSALL, D. M., 1989 - Paleoethnobotany. A Handbook of Procedures, 470p.; San Diego: Academic Press.

PEARSALL, D. M., 1992a - Prehistoric subsistence and agricultural evolution in the Jama River valley Manabí Province, Ecuador. Journal of the Steward Anthropological Society, 20 (N 1 y 2): 181-207.

PEARSALL, D. M., 1992b - The origins of plant cultivation in South America. In: The Origins of Agriculture: An International Perspective (C. Wesley Cowan \& P.J. Watson with the assistance of N.L. Benco, edit.): 173-205; Washington D.C. and London: Smithsonian Institution Press.

PEARSALL, D. M., 1993 - Contributions of phytolith analysis for reconstructing subsistence: examples from research in coastal Ecuador. In: Current Research in Phytolith Analysis: Applications in Archaeology and Paleoecology (D.M. Pearsall y D.R. Piperno, edit.): 109-122; MASCA, University of Pennsylvania Museum.

PEARSALL, D. M., 1994a - Issues in the analysis and interpretation of archaeological maize in South America. In: Corn and Culture in the Prehistoric New World (S. Johannesson y C. A. Hastorf, edit.): 245-272; Boulder: Westview Press.

PEARSALL, D. M., 1994b - Phytolith Analysis. In: Regional Archaeology in Northern Ecuador, Volume 1: Environment, Cultural Chronology and Prehistoric Subsistence in the Jama River Valley (J.A. Zeidler \& D. Pearsall, edit.): 161-174; Pittsburgh: University of Pittsburgh. Memoirs in Latin American Archaeology $\mathrm{N}^{\circ} 8$.

PEARSALL, D. M. \& PIPERNO, D. R., 1990 - Antiquity of maize cultivation in Ecuador: Summary and reevaluation of the evidence. American Antiquity, 55(2): 324-337.

PEARSALL, D. M. \& PIPERNO, D. R., 1993 - The nature and status of phytolith analysis. In: Current Research in Phytolith Analysis: Applications in Archaeology and Paleoecology (D.M. Pearsall \& D.R. Piperno, edit.): 9-18; Philadelphia, PA: MASCA, University of Pennsylvania Museum.

PIPERNO, D. R., 1984 - A comparison and differentiation of phytoliths of maize and wild grasses: Use of morphological criteria. American Antiquity, 49: 361-383.

PIPERNO, D. R., 1985 - Phytolith analysis and tropical paleo-ecology: Production and taxonomic significance of siliceous forms in New World plant domesticates and wild species. Review of Paleobotany and Palynology, 45: 185-228. 
PIPERNO, D. R., 1988 - Phytolith Analysis: An Archaeological and Geological Perspective; N.Y.: San Diego, Academic Press.

PIPERNO, D. R., 1990 - Aboriginal agriculture and land usage in the Amazon Basin, Ecuador. Journal of the Archaeological Science, 17: 665-677.

PIPERNO, D. R., 1991 - The status of phytolith analysis in the American tropics. Journal of World Prehistory, 5(2):155-191.

PIPERNO, D. R., 1994 - On the Emergence of Agriculture in the New World. Current Anthropology, 35(5): 637-643.

PIPERNO, D. R. \& PEARSALL, D. M., 1993 - Phytoliths in the reproductive structures of maize and teosinte: Implications for the study of maize evolution. Journal of Archaeological Science, 17: 665-677.

PIPERNO, D. R., CLAREY, K. H., COOKE, R. G., RANERE, A. J. \& WEILAND, D., 1985 Preceramic Maize in Central Panama. Phytolith, Pollen evidence. American Anthropologist, 87: 871-878.

RAPP, G. \& MULHOLLAND, S., 1992 - Phytolith Systematics: Emerging Issues; N.Y.: Plenum Press.

ROOSEVELT, A. C., 1984 - Problems interpreting the diffusion of cultivated plants. In: PreColumbian Plant Migration (D. Stone, edit.): 19-38; Harvard University Peabody Museum of Archaeology and Ethnology Papers, Volume 76. Papers Presented at the 44th International Congress of Americanists, Manchester, England.

ROVNER, I., 1971 - Potential of Opal Phytoliths for use in Paleoecological Reconstruction. Quaternary Research, 1: 343-359.

ROVNER, I., 1983 - Plant Opal Phytolith Analysis: Major Advances in Archaeobotanical Research. In: Advances in Archaeological Method and Theory Volume 6 (B. Schiffer, edit.): 225-266; N.Y.: Academic Press.

ROVNER, I., 1995 - "Mien, mean, and meaning. The limits of typology in phytolith analysis." Paper presented at the 60th Annual Meetings of the Society for American Archaeology, Minneapolis, Minn.

ROVNER, I., 1999-Phytolith Analysis. Response to Heather Pringle Special Section, Archaeology, Science, Volume 283(5401), 22 January 1999: 488-489.

ROVNER, I. \& RUSS, J., 1992 - Darwin and Design in Phytolith Systematics: Morphometric Methods for Mitigating Redundancy. In: Phytolith Systematics: Emerging Issues: 253276; New York: Plenum Press. Advances in Archaeological and Museum Science, Volume 1.

RUSS, J. C. \& ROVNER, I., 1989 - Stereological Identification of Opal Phytolith Populations from Wild and Cultivated Zea. American Antiquity, 54: 784-792.

SMITH, B. D., 1998 - Emergence of Agriculture, 231p.; Scientific American Library Publication. W. H. Freedman and Co.

SMITH, B. D., 1997 - Reconsidering the Ocampo Caves and the Era of Incipient Cultivation in Mesoamerica. Latin American Antiquity, 8(4): 342-383.

STAHL, P. W., 1984 - Tropical Forest Cosmology: The Cultural Context of Early Valdivia Occupations at Loma Alta. Ph.D. Dissertation, Department of Anthropology, University of Illinois, Urbana. University Microfilms International, Ann Arbor, 326p.

STALLER, J. E., 1992-1993 - El montículo ceremonial en el sitio de La Emerenciana en la costa sur del Ecuador y su significación del desarrollo de complejidad en la costa oeste de Sudamérica. Parte I. Cuadernos de Historia y Arqueología, 46-47: 14-37; Guayaquil, Ecuador: Publicación de la Casa la Cultura Ecuatoriana Benjamin Carrión, Nucleo del Guayas. 
STALLER, J. E., 1994 - Late Valdivia Occupation in El Oro Province Ecuador: Excavations at the Early Formative Period (3500-1500 B.C.) site of La Emerenciana. Ph.D. Dissertation, Department of Anthropology, Southern Methodist University Dallas, Texas. University Microfilms International, Ann Arbor, 526p.

STALLER, J. E., 1996 - El sitio Valdivia tardío de La Emerenciana en la costa sur del Ecuador y su significación del desarrollo de complejidad en la costa de sudamérica. Parte II. Cuadernos de Historia y Arqueología, 48-50: 65-118; Guayaquil, Ecuador: Publicación de la Casa la Cultura Ecuatoriana Benjamin Carrión, Nucleo del Guayas. Edición en homenaje a Olaf Holm.

STALLER, J. E. 2001 - The $\mathrm{Jel}^{\circ}$ Phase Complex at La Emerenciana, a late Valdivia site in southern El Oro Province, Ecuador. Andean Past, 6: 117-174. Cornell University, Latin American Studies Program.

THOMPSON, R. G., 1986 - "Opal Phytolith Analysis of Food Residues in Prehistoric Pottery and Plains Anthropological Problems." Paper Presented at the 46th Annual Plains Conference, Denver, Co.

THOMPSON, R. G., 1987a - "Research Potential of Opal Phytoliths Recovered from Food Residues in Utilized Ceramics." Paper Presented at the Second Phytolith Workshop. Columbia, Missouri.

THOMPSON, R. G., 1987b - Scanning Electron Microscopy Study of Opal Phytoliths Recovered from Food Residues in Utilized Ceramics at the Shea Site (32CS101). Report submitted to the Department of Sociology and Anthropology, Moorehead State University. (Ms.) on file. 0

THOMPSON, R. G. \& DOGAN, A. U., 1987 - The identification of corn in food residues on utilized ceramics. Journal of Electron Microscopy Techniques, 7(2): 146.

THOMPSON, R. G. \& MULHOLLAND, S., 1994 - The identification of corn in food residues on utilized ceramics at the Shea Site (32CS101). Phytolitharian Newsletter, 8(2): 7-11.

THOMPSON, R. G., \& STALLER, J. E., 2001 - An analysis of opal phytoliths from food residues of selected sherds and dental calculus from excavations at the site of La Emerenciana, El Oro Province, Ecuador. Phytolitherian, Bulletin of the Society for Phytolith Research, 13(2\&3): 8-16.

TYKOT, R. H., 1996 - "The dietary significance of prehistoric maize in the northern Andes." Paper presented at the 61st Annual Meetings of the Society for American Archaeology, New Orleans, Louisiana.

VAN DER MERWE, N., LEE-THORP, J. A. \& RAYMOND, J. S., 1993 - Light, Stable Isotopes and the Subsistence Base of Formative Cultures at Valdivia, Ecuador. In: Prehistoric Human Bone Archaeology at the Molecular Level (J. B. Lambert \& G. Grupe, edit.): 6397; Springer Verlag Ltd.

ZEVALLOS - MENENDEZ, C., 1971 - La agricultura en el formativo temprano del Ecuador (Cultura Valdivia); Guayaquil: Talleres gráficos de la Casa la Cultura Ecuatoriana, Nucleo del Guayas.

ZEVALLOS - MENENDEZ, C., GALINAT, W., LATHRAP, D. W., LENG, E. R., MARCOS, J. G. \& KLUMPP, K. M., 1977 - The San Pablo Corn Kernel and its friends. Science, 196: 385-389. 